\title{
Analytic studies of static and transport properties of (gauged) Skyrmions
}

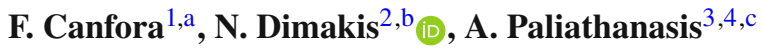 \\ ${ }^{1}$ Centro de Estudios Científicos (CECS), Casilla 1469, Valdivia, Chile \\ 2 Center for Theoretical Physics, College of Physical Science and Technology, Sichuan University, Chengdu 610065, China \\ ${ }^{3}$ Instituto de Ciencias Físicas y Matemáticas, Universidad Austral de Chile, Valdivia, Chile \\ ${ }^{4}$ Institute of Systems Science, Durban University of Technology, PO Box 1334, Durban 4000, Republic of South Africa
}

Received: 10 August 2018 / Accepted: 4 February 2019 / Published online: 14 February 2019

(C) The Author(s) 2019

\begin{abstract}
We study static and transport properties of Skyrmions living within a finite spatial volume in a flat (3+1)-dimensional spacetime. In particular, we derive an explicit analytic expression for the compression modulus corresponding to these Skyrmions living within a finite box and we show that such expression can produce a reasonable value. The gauged version of these solitons can be also considered. It is possible to analyze the order of magnitude of the contributions to the electrons conductivity associated to the interactions with this Baryonic environment. The typical order of magnitude for these contributions to conductivity can be compared with the experimental values of the conductivity of layers of Baryons.
\end{abstract}

\section{Introduction}

The appearance of Skyrme theory [1-3] disclosed very neatly the fundamental role of topology in high energy physics (see for instance [4-9]). First of all, the low energy QCD is very well described by the Skyrme theory $[10,11]$. Secondly, the solitons of this Bosonic theory (Skyrmions) describe Baryons. Thirdly, the Baryon charge is the winding number of the configuration (see [10-19] and references therein).

These arguments are more than enough to justify a profound analysis of the Skyrme model. Indeed, extensive studies of the latter can be found in literature (as the previous references clearly show). Not surprisingly, ${ }^{1}$ the Skyrme

\footnotetext{
${ }_{1}$ At least taking into account that it is reasonable to expect that the theory describing the low energy limit of QCD should be a quite complicated one.

a e-mail: canfora@cecs.cl

be-mail: nsdimakis@scu.edu.cn

c e-mail: anpaliat@phys.uoa.gr
}

field equations are a very hard nut to crack and, until very recently no analytic solution was available. Nevertheless, many numerical studies have shown that the Skyrme model provides results in good agreement with experiments.

Despite the success of the model and the existence of several solutions among different contexts, the analysis of their phenomenological aspects seldom can be carried out in an analytic manner. For an analytic solution and a relevant study in compact manifolds see [20].

The gauged Skyrme model (which describes the coupling of a $U(1)$ gauge field with the Skyrme theory) has also very important applications in the analysis of electromagnetic properties of Baryons, in the decay of nuclei in presence of defects (see [10,11,21-25] and references therein). Obviously, from the point of view of constructing analytic solutions, the $U$ (1) gauged Skyrme model is even worse than the original Skyrme theory. Until very recently, no explicit topologically non-trivial solution was available. Thus, topological configurations of this theory have been deeply analyzed numerically (see $[26,27]$ and references therein).

Here we list three relevant problems in the applications of (gauged) Skyrme theory to high energy phenomenology which will be the focus of the present paper.

(1) Finite density effects and the compression modulus: Finite density effects (and, in general, the phase diagrams) in the Skyrme model have been historically a very difficult topic to analyze with analytic methods. The lack of explicit solutions with topological charge living within a finite flat box with the spherical Skyrme ansatz is the origin of the problem. Some numerical results with the use of the spherical Skyrme ansatz are presented in [28-32] and references therein. Due to the fact that both finite volume effects and isospin chemical potential break spherical symmetry it is extremely difficult to improve the pioneering results in [28-32] without changing the original Skyrme ansatz. The main problem in this group 
is certainly the compression modulus [37-39] (to be defined precisely in the next section) which, roughly speaking, has to do with the derivative of the total energy of the Skyrmions with respect to the volume. The experimental value is different from the value derived using the original spherical hedgehog ansatz. The usual way to compute the compression modulus is to assume the Derrick rescaling for the reaction of nuclear matter to the action of external pressure (see the detailed discussion in [40]). The resulting value is higher than the experimental value. ${ }^{2}$ A closely related technical difficulty is that, if one uses the original hedgehog ansatz for the Skyrmion, it is very unclear even how to define the compression modulus since the original Skyrme ansatz describes a spherical Skyrmion living within an infinite volume so that to compute the derivatives of the energy with respect to the volume becomes a subtle question. The best way out of this difficulty would be, of course, to have a consistent ansatz for a Skyrmion living within a finite volume. Relevant numerical results in the literature on that problem are presented in [33-36] where non-spherical ansätze have been considered.

(2) Existence of Skyrmion-antiSkyrmion bound states/ resonances: Multi-Skyrmionic bound states of Baryon charge higher than 1 are known to exist and they have been successfully constructed numerically (see, for instance, [13] and references therein). However, until very recently, the problem of the existence of Skyrmion-antiSkyrmion bound states and resonances did not possess the place it deserved in the literature on the Skyrme model and despite its importance. We can refer to an early work on the subject in [41]. Here we shall study analytic results over the properties of such configurations. Experimentally, Baryon-antiBaryon bound states and resonances do exist [42-46]: these should correspond to Skyrmion-antiSkyrmion bound states. Such bound states are very difficult to find since the corresponding classical solutions are not static. Indeed, at a semi-classical level, Skyrmion-antiSkyrmion bound states should look like timeperiodic solutions in which a Skyrmion and an antiSkyrmion moves periodically around the center of mass of the system. These kinds of time-dependent configurations are difficult to analyze even numerically.

(3) Conductivities: The analysis of electrons transport through gauged Skyrmions is a very interesting open issue. At semi-classical level, one should solve the Dirac equation for the electron in the background of the gauged Skyrmion and, from the solution of the Dirac equation, one could compute the conductivity. It would be especially interesting to be able to describe complex structures assembled from neutrons

\footnotetext{
2 The following analysis suggests that this "uniform rescaling" assumption could be too strong. Indeed, the results at the end of Sect. 3 shows that Skyrme theory, when analyzed at finite density, provides with values of the compression modulus which are close to the experimental one.
}

and protons interacting with electromagnetic fields (such as slabs of Baryons interacting with the corresponding Maxwell field). In nuclear physics and astrophysics these structures are called nuclear pasta and they are very relevant in a huge variety of phenomena (see, for instance, [47-50] and references therein). On the other hand, there are very few "first principles" computations of the transport properties of these complex structures (see [51] and references therein). At a first glance, one could think that this kind of complex structure is beyond the reach of the gauged Skyrme model.

In order to achieve a deeper understanding of the above open issues, it is mandatory to be able to construct analytic examples of gauged multi-Skyrmionic configurations.

In [52-60] a strategy has been developed to generalize the usual spherical hedgehog ansatz to situations without spherical symmetry both in Skyrme and Yang-Mills theories (see [61-63] and references therein). Such a framework also allows to analyze configurations living within a finite region of space.

As far as the three open issues described above are concerned, this tool (which will be called here "generalized hedgehog ansatz") gave rise to the first derivation not only of the critical isospin chemical potential beyond which the Skyrmion living in the box ceases to exist, but also of the first explicit Skyrmion-antiSkyrmion bound states. Thus, this approach appears to be suitable to deal with the problems mentioned previously.

Interestingly enough, the generalized hedgehog ansatz can be adapted to the $U(1)$ gauged Skyrme model $[64,65]$ : it allowed the construction of two types of gauged solitons. Firstly, gauged Skyrmions living within a finite volume. Secondly, smooth solutions of the $U(1)$ gauged Skyrme model whose periodic time-dependence is protected by a topological conservation law (as they cannot be deformed to static solutions).

Here we demonstrate that by using this strategy it is possible to derive an explicit expression of the compression modulus. The transport properties of these gauged Skyrmions can also be analyzed. In this work we also present a simple estimate of the order of magnitude of the correction to the electron conductivities due to the interactions of the electrons with the baryonic environment. As far as transport properties are concerned, we will work at the level of approximation in which the electrons perceive the gauged Skyrmions as a classical background. Large $\mathbf{N}$ arguments strongly suggest that this is a very good approximation ${ }^{3}$ (see for a detailed

\footnotetext{
${ }^{3}$ In the leading 't Hooft approximation, in meson-Baryon scattering, the heavy Baryon (the Skyrmion in our case) is unaffected and, basically, only the meson can react. This is even more so in the electron-Baryon semiclassical interactions due to the huge mass difference between the Skyrmion and the electron. In this approximation, electrons perceive the Skyrmions as an effective medium.
} 
review chapter 4 and, in particular, section 4.2 of the classic reference [66]).

This paper is organized as follows: in Sect. 2 the action for the gauged Skyrme model and our notations will be introduced. In Sect. 3, the method to deal with Skyrmions at finite density will be described: as an application, a closed formula for the compression modulus of Skyrmions living within a cube will be derived. In Sect. 4, the gauged Skyrmions at finite density will be considered. In Sect. 5, the transport properties associated to electrons propagating in the Baryonic environment corresponding to the finite-density Skyrmions are analyzed. In Sect. 6, we draw some concluding ideas.

\section{The $U(1)$ gauged Skyrme model}

We consider the $U(1)$ gauged Skyrme model in four dimensions with global $S U(2)$ isospin internal symmetry and we will follow closely the conventions of $[64,65]$. The action of the system is

$$
\begin{gathered}
S=\int d^{4} x \sqrt{-g}\left[\frac { K } { 2 } \left(\frac{1}{2} \operatorname{Tr}\left(R^{\mu} R_{\mu}\right)\right.\right. \\
\left.\left.+\frac{\lambda}{16} \operatorname{Tr}\left(G_{\mu \nu} G^{\mu \nu}\right)\right)-\frac{1}{4} F_{\mu \nu} F^{\mu \nu}\right] \\
R_{\mu}=U^{-1} D_{\mu} U, \quad G_{\mu \nu}=\left[R_{\mu}, R_{\nu}\right] \\
D_{\mu}=\nabla_{\mu}+\kappa A_{\mu}\left[t_{3}, .\right]
\end{gathered}
$$

$U \in S U(2), \quad R_{\mu}=R_{\mu}^{j} t_{j}, \quad t_{j}=\dot{i} \sigma_{j}$,

where $\sqrt{-g}$ is the (square root of minus) the determinant of the metric, $F_{\mu \nu}=\partial_{\mu} A_{\nu}-\partial_{\nu} A_{\mu}$ is the electromagnetic field strength, $\nabla_{\mu}$ is the partial derivative, the positive parameters $K$ and $\lambda$ are fixed experimentally, $\kappa$ the coupling for the $U$ (1) field and $\sigma_{j}$ are the Pauli matrices. In our conventions $c=\hbar=\mu_{0}=1$, the space-time signature is $(-,+,+,+)$ and Greek indices run over space-time. The stress-energy tensor is

$$
\begin{aligned}
T_{\mu \nu}= & -\frac{K}{2} \operatorname{Tr}\left[R_{\mu} R_{\nu}-\frac{1}{2} g_{\mu \nu} R^{\alpha} R_{\alpha}\right. \\
& \left.+\frac{\lambda}{4}\left(g^{\alpha \beta} G_{\mu \alpha} G_{\nu \beta}-\frac{g_{\mu \nu}}{4} G_{\sigma \rho} G^{\sigma \rho}\right)\right]+\bar{T}_{\mu \nu},
\end{aligned}
$$

with

$$
\bar{T}_{\mu \nu}=F_{\mu \alpha} F_{\nu}^{\alpha}-\frac{1}{4} F_{\alpha \beta} F^{\alpha \beta} g_{\mu \nu}
$$

The field equations are

$$
\begin{aligned}
& D^{\mu}\left(R_{\mu}+\frac{\lambda}{4}\left[R^{v}, G_{\mu \nu}\right]\right)=0, \\
& \nabla_{\mu} F^{\mu \nu}=J^{v},
\end{aligned}
$$

where $J^{v}$ is the variation of the Skyrme action (the first two terms in Eq. (1)) with respect to $A_{v}$

$J^{\mu}=\frac{\kappa K}{2} \operatorname{Tr}\left[\widehat{O} R^{\mu}+\frac{\lambda}{4} \widehat{O}\left[R_{\nu}, G^{\mu \nu}\right]\right]$,

where

$\widehat{O}=U^{-1} t_{3} U-t_{3}$.

In the following sections, gauged Skyrmions and gauged time-crystals will be terms describing to the two different kinds of gauged topological solitons appearing as solutions of the coupled system expressed by Eqs. (5) and (6).

The aim of the present work is to show that the Skyrme model and its gauged version are able to give good predictions for important quantities such as the compression modulus and the conductivity.

\subsection{Topological charge}

The proper way to define the topological charge in the presence of a minimal coupling with a $U(1)$ gauge potential has been constructed in [21] (see also the pedagogical analysis in [26]):

$$
\begin{aligned}
W= & \frac{1}{24 \pi^{2}} \int_{\Sigma} \epsilon^{i j k} \operatorname{Tr}\left\{\left(U^{-1} \partial_{i} U\right)\left(U^{-1} \partial_{j} U\right)\left(U^{-1} \partial_{k} U\right)\right. \\
& \left.-\partial_{i}\left[3 \kappa A_{j} t_{3}\left(U^{-1} \partial_{k} U+\partial_{k} U U^{-1}\right)\right]\right\} .
\end{aligned}
$$

In the literature one usually only considers situations where $\Sigma$ is a space-like three-dimensional hypersurface. In these situations $W$ is the Baryon charge. In fact it has been recently shown $[64,65]$ that it is very interesting to also consider cases in which $\Sigma$ is time-like or light-like. Indeed, (whether $\Sigma$ is light-like, time-like or space-like) configurations with $W \neq 0$ cannot decay into the trivial vacuum $U=\mathbf{I}$. Hence, if one is able to construct configurations such that $W \neq 0$ along a time-like $\Sigma$, then the corresponding gauged soliton possesses a topologically protected timedependence as it cannot be continuously deformed into static solutions (since all the static solutions have $W=0$ along a time-like $\Sigma$ ). The natural name for these solitons is "(gauged) time-crystals" [64,65].

We can adopt the standard parametrization of the $S U(2)$ valued scalar $U\left(x^{\mu}\right)$

$U^{ \pm 1}\left(x^{\mu}\right)=Y^{0}\left(x^{\mu}\right) \mathbf{I} \pm Y^{i}\left(x^{\mu}\right) t_{i}, \quad\left(Y^{0}\right)^{2}+Y^{i} Y_{i}=1$

where $\mathbf{I}$ is the $2 \times 2$ identity and

$Y^{0}=\cos C, \quad Y^{i}=n^{i} \cdot \sin C$, 
$n^{1}=\sin F \sin G, \quad n^{2}=\sin F \cos G, \quad n^{3}=\cos F$,

with the help of which the standard baryon density (in the absence of a $U$ (1) field) reads $\rho_{B}=12 \sin ^{2} C \sin F d C \wedge$ $d F \wedge d G$. If we want a non-vanishing topological charge in this setting we have to demand $d C \wedge d F \wedge d G \neq 0$.

\section{Skyrmions at finite volume}

In the present section, the Skyrmions living within a finite flat box constructed in [64] will be slightly generalized. These explicit Skyrmionic configurations allow the explicit computations of the total energy of the system and, in particular, of its dependence on the Baryon charge and on the volume. Hence, among other things, one can arrive at a well-defined closed formula for the compression modulus.

The following ansatz for the representation of the $S U(2)$ group is the starting point of the analysis

$$
\begin{gathered}
G=\frac{q \phi-p \gamma}{2}, \quad \tan F=\frac{\tan H}{\sin A}, \\
\tan C=\tan A \sqrt{1+\tan ^{2} F},
\end{gathered}
$$

where

$A=\frac{p \gamma+q \phi}{2}, \quad H=H(r, z), \quad p, q \in \mathbb{N}$.

Moreover, it can be verified directly that, the topological density $\rho_{B}$ is non-vanishing. From the standard parametrization of $S U$ (2) [71] it follows that

$0 \leq \gamma \leq 4 \pi, \quad 0 \leq \phi \leq 2 \pi$

while the boundary condition for $H$ will be discussed below; in any case, its range is in the segment $H \in\left[0, \frac{\pi}{2}\right]$, while for $r$ we assume $0 \leq r \leq 2 \pi$. With the parametrization introduced by (12) and (13) the $S U$ (2) field assumes the form

$U= \pm\left(\begin{array}{cc}\cos (H) e^{\frac{1}{2} i(p \gamma+q \phi)} & \sin (H) e^{\frac{1}{2} i(p \gamma-q \phi)} \\ -\sin (H) e^{-\frac{1}{2} i(p \gamma-q \phi)} & \cos (H) e^{-\frac{1}{2} i(p \gamma+q \phi)}\end{array}\right)$.

Hereafter, we just consider the plus expression for $U$ throughout all the range of the variables $\gamma$ and $\phi$, which makes it a continuous function of the latter.

\subsection{Skyrmions in a rectangular cuboid}

We can extend the results presented in [64] by considering a cuboid with three different sizes along the three axis instead of a cube. Thus, we will use three - different in principle fundamental lengths characterizing each direction, $l_{1}, l_{2}$ and $l_{3}$, inside the metric.
The corresponding line element is

$d s^{2}=-d z^{2}+l_{1}^{2} d r^{2}+l_{2}^{2} d \gamma^{2}+l_{3}^{2} d \phi^{2}$.

The profile function that we consider depends only on one variable, ${ }^{4} H=H(r)$. We note that in this section we do not take into account the effects of an electromagnetic field, hence we have $A_{\mu}=0$ in the relations of the previous sections.

Under the aforementioned conditions the profile equation reduces to

$H^{\prime \prime}=\frac{\lambda l_{1}^{2} p^{2} q^{2}}{4\left(l_{2}^{2}\left(4 l_{3}^{2}+\lambda q^{2}\right)+\lambda l_{3}^{2} p^{2}\right)} \sin (4 H)$.

It is impressive that such a system, in flat space, can lead to an integrable equation for the profile. This is owed to the existence of a first integral of (17) that is given by

$$
\left(H^{\prime}\right)^{2}\left(l_{2}^{2}\left(4 l_{3}^{2}+\lambda q^{2}\right)+\lambda l_{3}^{2} p^{2}\right)+\frac{\lambda l_{1}^{2} p^{2} q^{2}}{8} \cos (4 H)=I_{0}
$$

The above relation can be written as

$\left(\tilde{H}^{\prime}\right)^{2}-k \sin (\tilde{H})^{2}=\tilde{I}_{0}$,

where

$$
\begin{gathered}
\tilde{H}=2 H, \quad k=\frac{\lambda l_{1}^{2} p^{2} q^{2}}{l_{2}^{2}\left(4 l_{3}^{2}+\lambda q^{2}\right)+\lambda l_{3}^{2} p^{2}}, \\
\tilde{I}_{0}=\frac{8 I_{0}-\lambda l_{1}^{2} p^{2} q^{2}}{8 l_{2}^{2} l_{3}^{2}+2 \lambda l_{2}^{2} q^{2}+2 \lambda l_{3}^{2} p^{2}} .
\end{gathered}
$$

Subsequently, we can bring (46) into the form

$$
\frac{d \tilde{H}}{d r}= \pm \sqrt{\tilde{I}_{0}}\left(1-\tilde{k}(\sin \tilde{H})^{2}\right)^{\frac{1}{2}}
$$

where we have set $\tilde{k}=-k / \tilde{I}_{0}$. The last expression leads to

$$
\sqrt{\tilde{I}_{0}} \int_{0}^{r} d \bar{r}= \pm \int_{0}^{\tilde{H}}\left(1-\tilde{k}(\sin \bar{H})^{2}\right)^{-\frac{1}{2}} d \bar{H}
$$

where we have introduced the bars in order to distinguish the variables that are integrated from the $r$ and $\tilde{H}(r)$ which are the boundaries of the two integrals. Of course we consider $\tilde{I}_{0}>0$. As a starting point for the integration we take $r=0$, $\tilde{H}(0)=0=H(0)$, although we could also set $r=0, \tilde{H}=$ $\pi\left(H(0)=\frac{\pi}{2}\right)$. The difference between the two boundary

\footnotetext{
${ }^{4}$ On the other hand, when the coupling with Maxwell field is neglected, the profile can depend on time as well. In this case, one gets an effective sine-Gordon theory for the profile $H(t, r)$ [64].
} 
choices is just in the sign of the topological charge. These boundary values, for $H$ and those that we have seen in (14) for $\gamma$ and $\phi$ lead to a topological charge $W=p q$ in (8) (for $\left.A_{\mu}=0\right)$.

In the right hand side of (22) we recognize the incomplete elliptic integral defined as

$F(\tilde{H} \mid \tilde{k})=\int_{0}^{\tilde{H}}\left(1-\tilde{k}(\sin \bar{H})^{2}\right)^{-\frac{1}{2}} d \bar{H}$

The solution to the differential equation (47) is just the inverse of this function, which is called the Jacobi amplitude am = $F^{-1}(\tilde{H} \mid \tilde{k})$. So, in terms of our original Eq. (17) the solution reads

$H(r)= \pm \frac{1}{2} \operatorname{am}\left(\tilde{I}_{0}^{1 / 2} r \mid \tilde{k}\right)$

Finally, by considering the positive branch, the value of the constant of integration $\tilde{I}_{0}$ is governed by the boundary condition $H(2 \pi)=\frac{\pi}{2}$.

In the special case when $l_{1}=l_{2}=l_{3}=l$ we obtain the particular case which was studied in [64]. Here, we give emphasis to this general case and, especially, we want to study the most energetically convenient configurations and the way in which they are affected by the anisotropy in the three spatial directions. In Fig. 1 we see a schematic representation of the finite box we are considering for this Skyrmionic configuration with a baryon number $B=p q$.

The physical configuration that we try to reproduce with this model is the structure of matter in nuclear pasta. The latter is a dense form of matter that is encountered inside the crusts of neutron stars. Thus, we make this "crude" (but analytic in its results) model trying to imitate with these $p$ and $q$ Skyrmionic layers a particular form of this matter that is encountered in nature. The dimensions of the configuration are governed by the three numbers $l_{1}, l_{2}$ and $l_{3}$. Of course we do not expect the binding energies of such a configuration to be at the same level with those produced by the usual spherically symmetric ansatz. This is something that we examine thoroughly in the next section.

\subsubsection{The energy function}

We proceed to study the energy function for the solution that we previously introduced. The constant of motion $I_{0}$ in (18) can be expressed in terms of the other constants of the model if we consider the boundary values $H(0)=0$ and $H(2 \pi)=\pi / 2$. By solving (18) with respect to $H^{\prime}$ and integrating the resulting relation with respect to $r$ we obtain

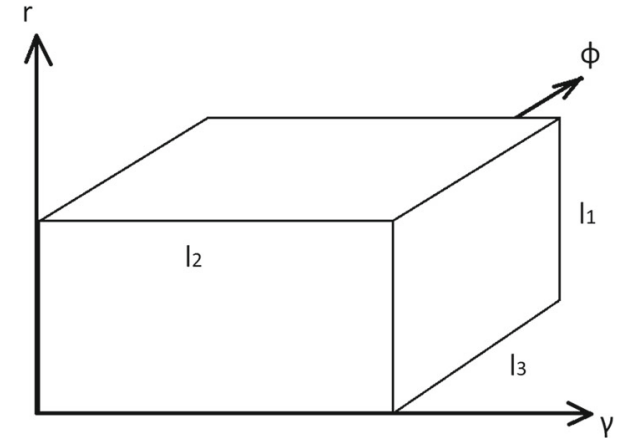

Fig. 1 The finite box of the Skyrmionic system

$2 \sqrt{2} \int_{a}^{b}\left(\frac{l_{2}^{2}\left(4 l_{3}^{2}+q^{2}\right)+l_{3}^{2} p^{2}}{8 I_{0}-l_{1}^{2} p^{2} q^{2} \cos (4 H)}\right)^{1 / 2} d H=\int_{0}^{2 \pi} d r$

which leads to

$l_{1}=\frac{x \mathrm{~K}\left(-x^{2}\right) \sqrt{l_{2}^{2}\left(4 l_{3}^{2}+q^{2}\right)+l_{3}^{2} p^{2}}}{\pi p q}$,

where $\mathrm{K}$ is the complete elliptic integral of the first kind and $x$ is related to $I_{0}$ through

$I_{0}=\frac{l_{1}^{2} p^{2} q^{2}\left(x^{2}+2\right)}{8 x^{2}}$

The pure time component of the energy momentum tensor in our case is

$$
\begin{aligned}
T_{00}= & \frac{K}{8 V^{2}}\left[\left(l_{2}^{2}\left(4 l_{3}^{2}+\lambda q^{2}\right)+\lambda l_{3}^{2} p^{2}\right) H^{\prime 2}\right. \\
& \left.+\frac{\lambda l_{1}^{2} p^{2} q^{2}}{4} \sin ^{2}(2 H)+V^{2}\left(\frac{p^{2}}{l_{2}^{2}}+\frac{q^{2}}{l_{3}^{2}}\right)\right] .
\end{aligned}
$$

As a result we can calculate the energy from the expression

$E=\int_{\Sigma} \sqrt{-{ }^{(3)} g} T_{00} d^{3} x=8 \pi^{2} V \int_{0}^{\frac{\pi}{2}} \frac{T_{00}}{H^{\prime}} d H$.

We can write the integrand as a pure function of $H$ with the help of (18) and obtain - in principle - the energy as a function of the $l_{i}$ 's, $p$ and $q$. However, due to the fact that relation (26) cannot be straightforwardly inverted so as to substitute $I_{0}$ as a function of $l_{1}$ (through (26) and (27)) we choose to express the energy function in terms of $x$ instead of $l_{1}$. In what follows, we assume the values $K=2$ and $\lambda=1$ for the coupling constants [14], so that lengths are measured in $\mathrm{fm}$ and the energy in $\mathrm{MeV}$. In this manner we get 


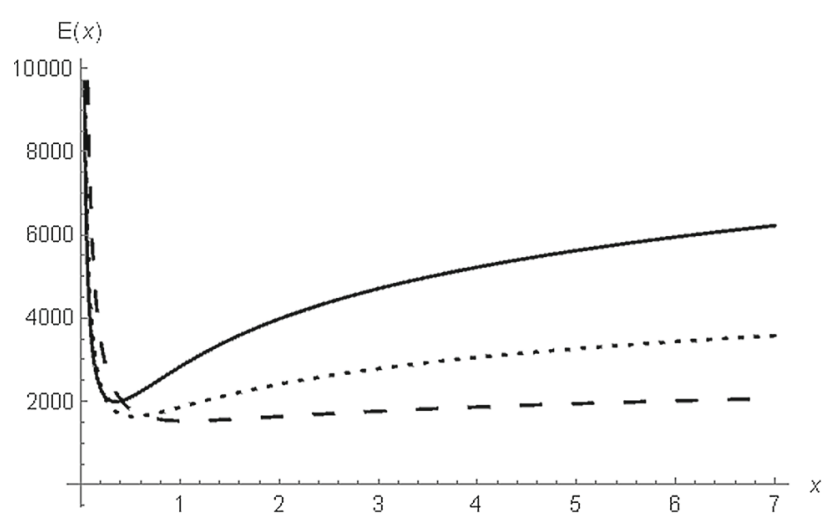

Fig. 2 The plots of $E(x)$ (in $\mathrm{MeV}$ ) for three sets of values: (a) $p=$ $q=3, l_{2}=l_{3}=1 \mathrm{fm}$ (dashed line), (b) $p=q=3, l_{2}=l_{3}=2 \mathrm{fm}$ (dotted line) and (c) $p=q=3, l_{2}=l_{3}=3 \mathrm{fm}$ (continuous line). The minimum of the energy corresponds to $l_{1}=0.227 \mathrm{fm}, l_{1}=0.323 \mathrm{fm}$ and $l_{1}=0.42 \mathrm{fm}$ respectively

$$
\begin{aligned}
& E\left(x, l_{2}, l_{3}, p, q\right) \\
& =\frac{\pi^{2} p q \sqrt{l_{2}^{2}\left(4 l_{3}^{2}+q^{2}\right)+l_{3}^{2} p^{2}}}{l_{2} l_{3}} \\
& \quad \times \frac{K\left(-x^{2}\right)\left(\frac{4 l_{2}^{2} x^{2} K\left(-x^{2}\right)}{p^{2}}-\frac{K\left(-x^{2}\right)\left(q^{2}-4 l_{3}^{2} x^{2}\right)}{q^{2}}+2 \mathcal{E}\left(-x^{2}\right)\right)}{x\left|K\left(-x^{2}\right)\right|},
\end{aligned}
$$

where $\mathcal{E}$ is the complete elliptic integral of the second kind. The $x$, as we discussed, is linked - with the help of the boundary conditions of the problem - through (26) to $l_{1}$. If we fix all variables apart from $x$ and plot the energy as a function of the latter we get what we see in Fig. 2. In this graph, we observe that the minimum of the energy is "moving" to smaller values of $x$ as the box is being enlarged in the two directions of $l_{2}$ and $l_{3}$. However, we have to keep in mind that the other of the lengths, namely $l_{1}$, depends also on the values of $l_{2}$ and $l_{3}$ through (26). For the particular set of values used in the figure we can see that as $l_{2}$ and $l_{3}$ rise, $l_{1}$ is also relocated to larger values. In the next section we study more thoroughly the function $E\left(x, l_{2}, l_{3}, p, q\right)$ and its derivatives near the values that correspond to the most energetically convenient configurations.

\subsubsection{The energy as a function of the three $l_{i}$ 's}

Let us see how the energy behaves in terms of the three fundamental lengths $l_{1}, l_{2}$ and $l_{3}$ under the condition that we fix $p$ and $q$ to specific values. In the Table 1 we can observe the location of the minimum of the energy for specific values of $p$ and $q$.

First, we have to note that the interchange of $p$ and $q$ makes no significant difference, so weather you take $p=100$ and $q=50$ or $p=50$ and $q=100$, the only thing that hap-
Table 1 Minimum of the energy for values of $p$ and $q$

\begin{tabular}{lrrlrr}
\hline$E_{\text {min }}(\mathrm{MeV})$ & $p$ & $q$ & $l_{1}(\mathrm{fm})$ & $l_{2}(\mathrm{fm})$ & $l_{3}(\mathrm{fm})$ \\
\hline 167 & 1 & 1 & 0.251 & 0.413 & 0.413 \\
334 & 1 & 2 & 0.251 & 0.413 & 0.826 \\
669 & 2 & 2 & 0.251 & 0.826 & 0.826 \\
835638 & 100 & 50 & 0.251 & 41.306 & 20.653 \\
835638 & 50 & 100 & 0.251 & 20.653 & 41.306 \\
\hline
\end{tabular}

pens is that the values of the corresponding lengths $l_{2}$ and $l_{3}$ are also interchanged. However, the arithmetic value that the energy assumes remains the same. Another thing that we have to notice is that, if we calculate the percentage difference of the minimum of the energy from the topological bound $E_{0}=12 \pi^{2}|B|=12 \pi^{2} p q$; in all cases we get $\Delta(\%)=\frac{E-E_{0}}{E_{0}}(\%)=41.11 \%$. Thus, we see that the minimum of the energy $E\left(l_{1}, l_{2}, l_{3}\right)$ has a fixed deviation from the Bogomol'nyi bound irrespectively of the $p, q$ configuration. We also observe that this most energetically convenient situation arises when the box has convenient lengths. In particular we see that the relation $\frac{l_{2}}{l_{3}}=\frac{p}{q}$ is satisfied in all cases, while $l_{1}$ remains fixed in a single "optimal" value. By comparing with the usual spherically symmetry Skyrmionic configuration in an infinite volume, this higher deviation from the Bogomol'nyi bound may be anticipated due to the "compression" of the system into a finite volume.

It is also interesting to study the first derivatives of the energy with respect to the three lengths of the box. To this end, and since we have $E$ in terms of $x$ which also involves $l_{1}, l_{2}$ and $l_{3}$ we need to write

$$
\begin{aligned}
d E\left(x, l_{2}, l_{3}\right)= & \frac{\partial E}{\partial x} d x+\frac{\partial E}{\partial l_{2}} d l_{2}+\frac{\partial E}{\partial l_{3}} d l_{3} \\
= & \frac{\partial E}{\partial x} \frac{\partial x}{\partial l_{1}} d l_{1}+\left(\frac{\partial E}{\partial x} \frac{\partial x}{\partial l_{2}}+\frac{\partial E}{\partial l_{2}}\right) d l_{2} \\
& +\left(\frac{\partial E}{\partial x} \frac{\partial x}{\partial l_{3}}+\frac{\partial E}{\partial l_{3}}\right) \\
= & d \tilde{E}\left(l_{1}, l_{2}, l_{3}\right) .
\end{aligned}
$$

In Fig. 3 we can see the general behavior of three $\frac{\partial \tilde{E}}{\partial l_{i}}$ for fixed $l_{1}=0.251$ in terms of $l_{2}$ and $l_{3}$ near the values where the energy assumes its minimum. On the other hand, in Fig. 4 we plot the derivatives of the energy with respect to $x$ after fixing $l_{2}$ and $l_{3}$ to their minimum value for various $p, q$ configurations. We can see that $\frac{\partial E}{\partial l_{2}}$ and $\frac{\partial E}{\partial l_{3}}$ are indistinguishable when $p=q$. On the other hand if $q>p$ the $\frac{\partial E}{\partial l_{3}}$ line runs closer to the vertical axis than $\frac{\partial E}{\partial l_{2}}$ and vice versa when $p>q$. Finally, before proceeding to study the energy as a function of $p$ and $q$, we give in Fig. 5 its graph in terms of $l_{2}$ and $l_{3}$ when $l_{1}$ assumes the value that corresponds to the minimum of the energy. 


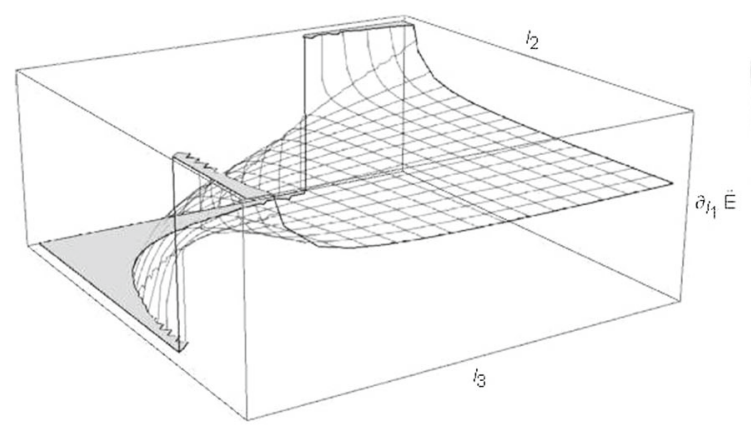

(a) Derivative of the energy with respect to $l_{1}$

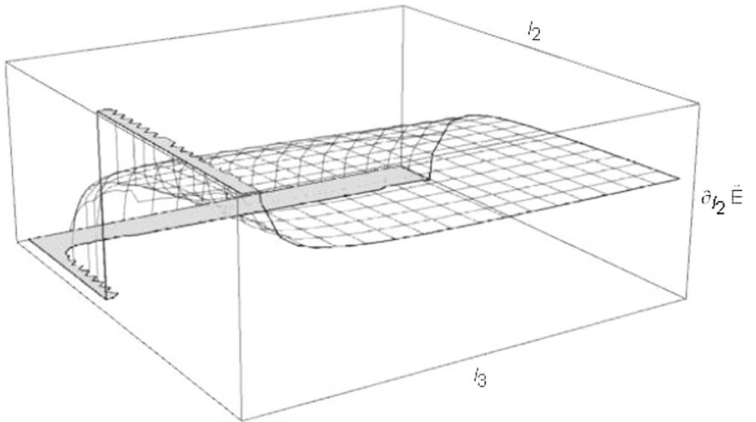

(b) Derivative of the energy with respect to $l_{2}$

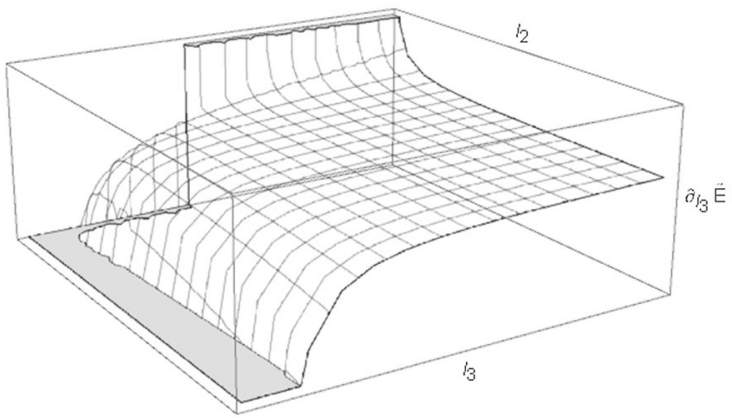

(c) Derivative of the energy with respect to $l_{3}$

Fig. 3 Derivative of the energy, in terms of the basic dimensions of the Skyrmionic box, near its minimum value. The behaviour of the three $\frac{\partial E}{\partial l_{i}}$ is the same irrespectively of $p$ and $q$. The only thing that changes

\subsection{The energy of the symmetric configuration}

Due to using (26) in the previous section so as to write the energy as a function of $x, l_{2}$ and $l_{3}$, it is not straightforward from that expression to derive what happens in the case where one considers a symmetric box $l_{1}=l_{2}=l_{3}=l$. In this section we treat this situation from the very beginning by setting all fundamental lengths as equal in Eq. (18). We have to note that throughout this section we also make use of the system of units $K=2, \lambda=1$. The expression relative to (25), from the resulting integral of motion, leads to

$l=\frac{\sqrt{\pi^{2} p^{2} q^{2}-x^{2} \mathrm{~K}\left(-x^{2}\right)^{2}\left(p^{2}+q^{2}\right)}}{2 x \mathrm{~K}\left(-x^{2}\right)}$

where $x$ is defined as in the previous section by relation (27), with $l_{1}=l$. By following the exact same steps as before we are led to the following expression for the energy is the scaling of the figures since $l_{2}$ and $l_{3}$ and $\frac{\partial E}{\partial l_{i}}$ assume larger values as $p$ and $q$ increase

is easy to note that the energy is symmetric under the mirror change $p \leftrightarrow q$. We verify that the for a bigger baryon number, the most optimal configuration corresponds also to a larger box. In Fig. 6 we can see the plot of the energy with respect to various configurations demonstrating the aforementioned fact. The second thing that we can note is that the deviation $\Delta=\frac{E-E_{0}}{E_{0}}$ from saturating the bound also increases for larger baryonic configurations. In Table 2 we provide some basic examples. Surprisingly we can see that the configuration $p=q=2$ is slightly more convenient than the one corresponding to $p=2, q=1$. As long as we know, this is the only case where this is happening. In general it can be seen that the $p=q$ construction requires more energy than the $p, q-1$, with an exception in the $p=q=2$ case.

\subsection{The compression modulus for the rectangular box}

From the technical point of view, it is worth to emphasize here that the very notion of compression modulus would require to put the Skyrmions within a finite flat box of volume $V$ : then the compression modulus is related to the second derivative of

$E_{c}(x, p, q)=\frac{2 \pi^{3}\left(2 p^{2} q^{2} \mathrm{~K}\left(-x^{2}\right) \mathcal{E}\left(-x^{2}\right)-\mathrm{K}\left(-x^{2}\right)^{2}\left(p^{4} x^{2}+p^{2} q^{2}\left(2 x^{2}+1\right)+q^{4} x^{2}\right)+\pi^{2} p^{2} q^{2}\left(p^{2}+q^{2}\right)\right)}{x^{2} \mathrm{~K}\left(-x^{2}\right) \sqrt{\frac{\pi^{2} p^{2} q^{2}}{x^{2}}-\mathrm{K}\left(-x^{2}\right)^{2}\left(p^{2}+q^{2}\right)}}$. 


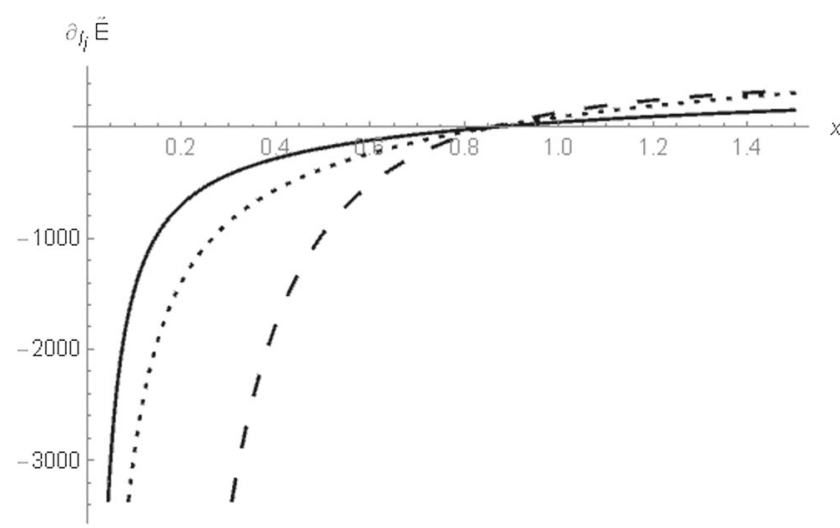

(a) $\frac{\partial E}{\partial l_{i}}$ for $p=1, q=2$

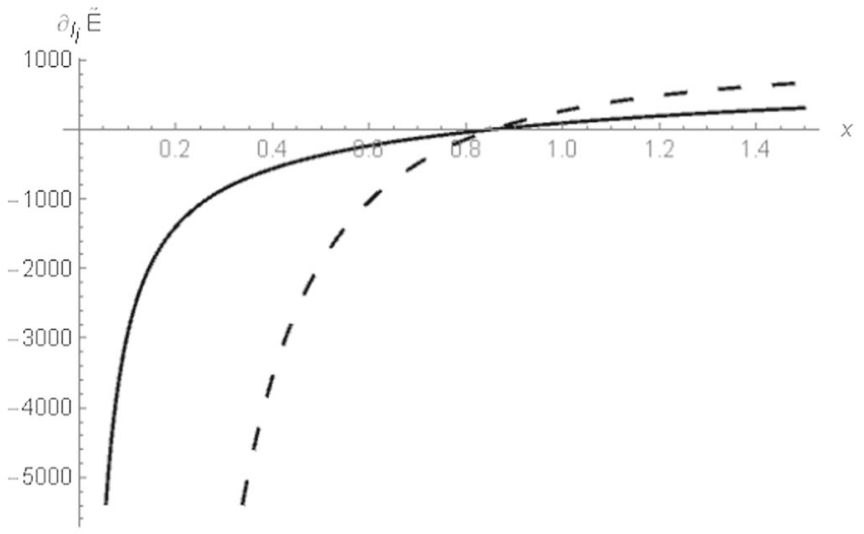

(b) $\frac{\partial E}{\partial l_{i}}$ for $p=2, q=2$

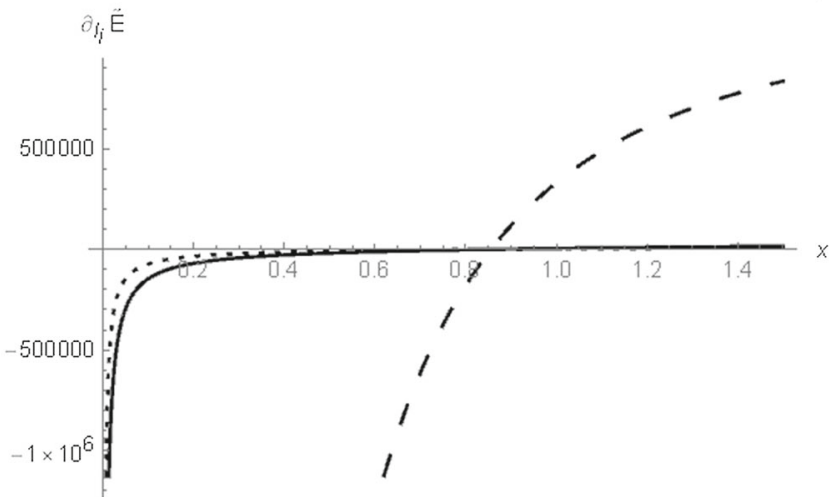

(c) $\frac{\partial E}{\partial l_{i}}$ for $p=100, q=50$

Fig. 4 Derivative of the energy with respect to the $l_{i}$ 's given as function of $x$. In every case the dashed line corresponds to $\frac{\partial E}{\partial l_{1}}$, the dotted to $\frac{\partial E}{\partial l_{2}}$ and the continuous line to $\frac{\partial E}{\partial l_{3}}$. Lengths are measured in fm and the energy in $\mathrm{MeV}$

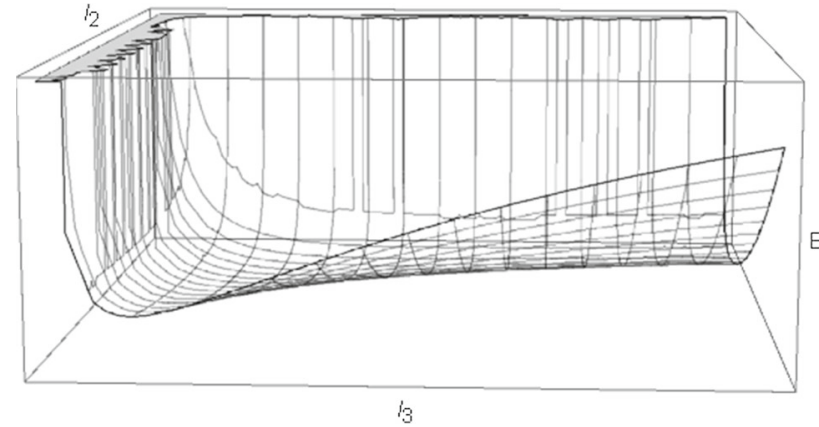

Fig. 5 Plot of the energy $E$ in the $l_{2}-l_{3}$ plane when $l_{1}$ takes the value that corresponds to the minimum of $E$

the total energy of the system with respect to $V$. As it has been already mentioned, this requires to generalize the hedgehog ansatz to situations without spherical symmetry. On the other hand, if one insists in defining the compression modulus for the spherical hedgehog, it becomes a rather subtle issue (see the nice analysis in [40]) how to define the derivative of the energy with respect to the volume. Here we are using the generalized hedgehog ansatz $[64,65]$ which is well suited

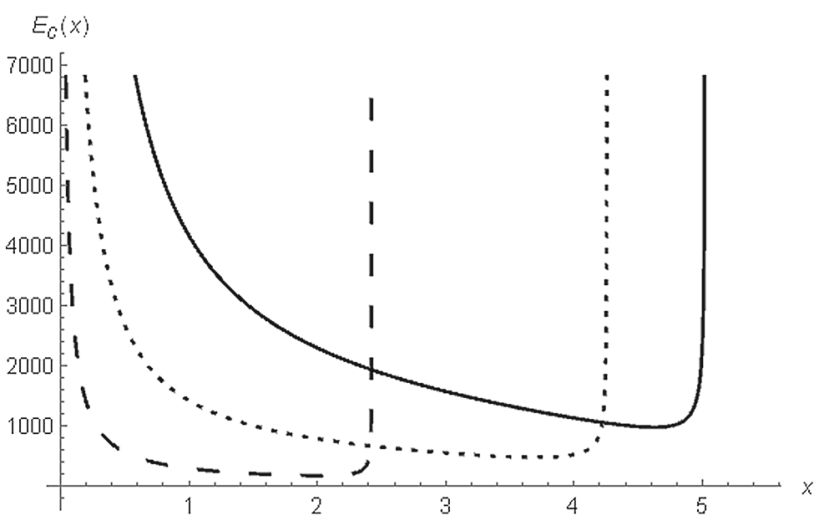

Fig. 6 Plot of the energy of the cubic configuration $E_{c}$ with respect to $x$. The dashed line corresponds to $p=q=1$, the dotted to $p=2$, $q=1$ and the continuous line to $p=3, q=1$. The minimum of the energy in terms of the size of the cube $l$ is: $l=0.322, l=0.369$ and $l=0.385$ respectively

to deal with situations without spherical symmetry. In this way we can analyze Skyrmions living within a region of flat space-time of finite spatial volume avoiding all the sub- 
Table 2 Deviation from the topological bound for several values of $p$ and $q$

\begin{tabular}{rrrr}
\hline$p$ & $q$ & $l(\mathrm{fm})$ & $\Delta(\%)$ \\
\hline 1 & 1 & 0.322 & 53 \\
2 & 1 & 0.369 & 105 \\
3 & 1 & 0.385 & 177 \\
2 & 2 & 0.463 & 104 \\
3 & 2 & 0.505 & 138 \\
3 & 3 & 0.571 & 148 \\
\hline
\end{tabular}

tleties mentioned above. In particular, in the present case the "derivative with respect to the volume" means, literally, the derivative (of the total energy of the system) with respect to the spatial volume of the region in which the Skyrmions are living.

As we obtained the general behavior of the three $\frac{\partial E}{\partial l_{i}}$ functions in the previous sub-sections, we are also able to derive an analytic expression of the compression modulus $[72,73]$

$\mathcal{K}=\frac{9 V}{B \beta} \approx 210 \pm 30 \mathrm{MeV}$

where $\beta=-\frac{1}{V} \frac{\partial V}{\partial P}$ is the compressibility. By using $P=\frac{d E}{d V}$ we acquire

$\mathcal{K}=-\frac{9 V^{2}}{B} \frac{d^{2} E}{d V^{2}}$,

where $B$ is the baryon charge and $V$ the finite volume in which we confine the system; in our case this volume is $V=16 \pi^{3} l_{1} l_{2} l_{3}$. The difference in the sign of (34) in comparison to other expressions in the literature [74] is owed to the metric signature that we follow here and which affects the derivation of $E$ from $T_{00}$. In order to express the energy that we obtain from (30) as a function of the volume, we introduce the following reparametrization of the $l_{i}$ 's into three new variables

$$
\begin{aligned}
& l_{1}=c_{1}\left(\frac{V}{16 \pi^{3}}\right)^{1 / 3}, \quad l_{2}=c_{2}\left(\frac{V}{16 \pi^{3}}\right)^{1 / 3}, \text { and } \\
& l_{3}=\frac{1}{c_{1} c_{2}}\left(\frac{V}{16 \pi^{3}}\right)^{1 / 3},
\end{aligned}
$$

so that $l_{1} l_{2} l_{3}=\frac{V}{16 \pi^{3}}$. We can substitute the above expressions into both (26) and (30). By solving the first with respect to $V$ and substituting to the second we obtain the energy as a pure function of $x$ which is associated through (26) with the volume $V$. We can thus calculate the first and second derivatives of the energy with respect to the volume by just taking $\frac{d E}{d V}=\left(\frac{d V}{d x}\right)^{-1} \frac{d E}{d x}$ and $\frac{d^{2} E}{d V^{2}}=\left(\frac{d V}{d x}\right)^{-1} \frac{d}{d x}\left[\left(\frac{d V}{d x}\right)^{-1} \frac{d E}{d x}\right]$.

The first derivative of $E(V)$ with respect to the volume defines the pressure of the system, i.e. $P=\frac{d E}{d V}$. In Fig. 7 we see the graphs of the pressure the compression modulus and the energy with respect to the volume for specific regions of the variable $V$. Due to the complicated nature of the relation between $x$ and $V$ it is not easy to put in this parametric plot the behavior of $P$ and $E$ near the region where $V \rightarrow 0$. However, one can calculate through the relations that as one shrinks the volume to zero, the pressure suddenly falls and changes sign becoming negative. The same happens to the compression modulus $\mathcal{K}$ as well, for even smaller values of $V$, while the energy remains positive for all $V$. Unfortunately the expressions are too cumbersome to present them analytically in this work, but the graphs in Fig. 7 demonstrate the general behavior. In the case of a finite cube with $l_{1}=l_{2}=l_{3}$ the situation is a lot simpler as we can see in the following section.

\subsubsection{Compression modulus in the symmetric case}

The most natural case corresponds to choose $l_{1}=l_{2}=l_{3}=$ $l$. In this way, we can derive a closed analytic formula for the compression modulus of the Skyrmions living within such a cuboid. To the best of our knowledge, this is the first case in which one can derive an analytic formula (Eqs. (36) and (37) below) for the compression modulus in a highly interacting theory such as the low energy limit of QCD. Indeed, by expressing the fundamental length as $l=\left(\frac{V}{16 \pi^{3}}\right)^{1 / 3}$ we can easily use (32) to relate the volume $V$ with the variable $x$ on which the energy depends (33). In this manner we can get an analytical expression for the compression modulus of the cube in terms of the variable $x$, which is

$$
\begin{aligned}
\mathcal{K}(x)= & -\frac{36}{p q}\left[\left(x^{2}+1\right) \mathrm{K}\left(-x^{2}\right)^{3}\right. \\
& \times\left(\pi^{2} p^{2} q^{2}-x^{2} \mathrm{~K}\left(-x^{2}\right)^{2}\left(p^{2}+q^{2}\right)\right)^{2} \\
& +x^{2} \mathrm{~K}\left(-x^{2}\right)^{3}\left(p^{2}+q^{2}\right) \mathcal{E}\left(-x^{2}\right)^{2} \\
& \times\left(5 \pi^{2} p^{2} q^{2}-x^{2} \mathrm{~K}\left(-x^{2}\right)^{2}\left(p^{2}+q^{2}\right)\right) \\
& +\pi^{4} p^{2} q^{2} \mathcal{E}\left(-x^{2}\right)\left(\mathrm{K}\left(-x^{2}\right)^{2}\right. \\
& \times\left(x^{2}\left(p^{2}+q^{2}\right)^{2}-2 p^{2} q^{2}\right) \\
& \left.\left.-\pi^{2} p^{2} q^{2}\left(p^{2}+q^{2}\right)\right)\right] .
\end{aligned}
$$

It can be shown that the parametric plots with respect to the volume which is

$V=2 \pi^{3} \frac{\left(\pi^{2} p^{2} q^{2}-x^{2} \mathrm{~K}\left(-x^{2}\right)^{2}\left(p^{2}+q^{2}\right)\right)^{3 / 2}}{x^{3} \mathrm{~K}\left(-x^{2}\right)^{3}}$

lead to the same behavior for the pressure, the energy and the compression modulus that has being derived in the previous section. For various values of $p$ and $q$ the behavior of the before mentioned quantities is described by the same graphs as given in Fig. 7.

A baryon density $\left(n=\frac{B}{V}\right.$ ) of $0.04 \mathrm{fm}^{-3} \lesssim n \lesssim 0.07 \mathrm{fm}^{-3}$ is assumed [75] to be appropriate for characterizing nuclear pasta and in particular lasagna. Within this range densities we 
Fig. 7 Parametric plots of the pressure $P$, the compression modulus $\mathcal{K}$ and the energy $E$ with respect to the volume. The plots correspond to the same parameters but for different ranges of the volume

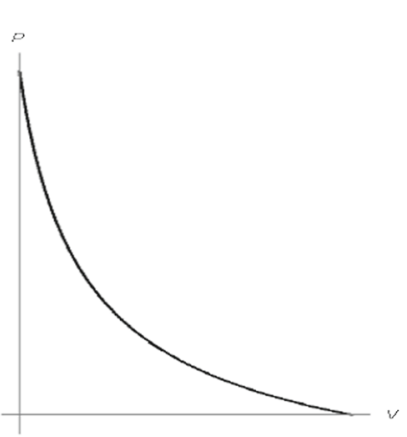

(a) Pressure $P(V)$

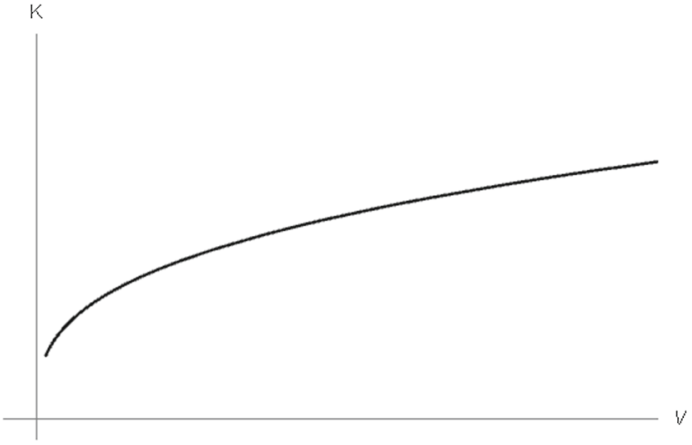

(b) Compression modulus $\mathcal{K}(V)$

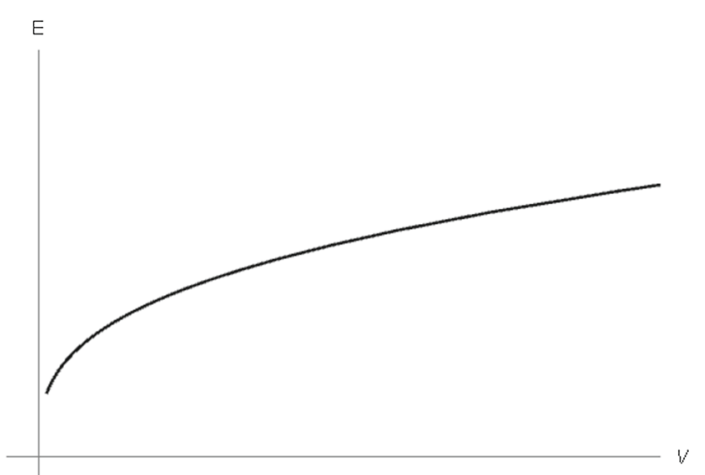

(c) Energy $E(V)$

Table 3 Examples of configurations corresponding to a compression modulus $\mathcal{K} \sim 230 \mathrm{MeV}$

\begin{tabular}{lllll}
\hline$B$ & 144 & 196 & 225 & 324 \\
\hline$n\left(\mathrm{fm}^{-3}\right)$ & 0.044 & 0.048 & 0.051 & 0.057 \\
\hline
\end{tabular}

can see that with expressions (36) and (37) we can achieve a compression modulus around $\mathcal{K} \sim 230 \mathrm{MeV}$ (which is quite reasonable [40,76]). For instance in Table 3 one can observe various examples of configurations involving baryon densities $n$ and the corresponding baryon numbers $B$, whose compression modulus - as calculated with the help of (36) is $\mathcal{K} \sim 230 \mathrm{MeV}$. In all cases presented in the table we have considered $p=q$, thus $B=p^{2}$.

\section{Gauged solitons}

Here we will shortly describe (a slight generalization of) the gauged solitons constructed in [65].

\subsection{Gauged Skyrmions}

As in [65], we introduce an electromagnetic potential of the form

$$
A_{\mu}=\left(b_{1}(r), 0, b_{2}(r), b_{3}(r)\right),
$$

to be coupled to the multi-Skyrmionic system under consideration. The Maxwell equations (6) reduce to

$b_{i}^{\prime \prime}=\kappa^{2} M_{i j} b_{j}+\kappa N_{i}$

with the nonzero components of $M$ and $N$ being

$$
\begin{aligned}
M_{11}= & -K \sin ^{2}(H) \\
& \times\left[l_{1}^{2}\left(4+\lambda\left(\frac{p^{2}}{l_{2}^{2}}+\frac{q^{2}}{l_{3}^{2}}\right) \cos ^{2}(H)\right)+4 \lambda H^{\prime 2}\right] \\
M_{23}= & \frac{K \lambda l_{1}^{2} p q}{4 l_{3}^{2}} \sin ^{2}(2 H) \\
M_{32}= & \frac{l_{3}^{2}}{l_{2}^{2}} M_{23} \\
M_{22}= & M_{11}+\frac{p}{q} M_{32} \\
M_{22}= & M_{11}+\frac{q}{p} M_{23} \\
N_{2}= & \frac{p}{4} M_{11}+\frac{1}{4}\left(\frac{l_{3}^{2} p^{2}}{l_{2}^{2} q}-q\right) M_{23} \\
N_{3}= & -\frac{q}{4} M_{11}-\frac{1}{4}\left(\frac{l_{2}^{2} q^{2}}{l_{3}^{2} p}-p\right) M_{32} .
\end{aligned}
$$

A direct computation shows that, using the line element in Eq. (16), the three coupled gauged Skyrme equations (namely, $E^{j}=0, j=1,2,3$ ) in Eq. (5) 
$D^{\mu}\left(R_{\mu}+\frac{\lambda}{4}\left[R^{\nu}, G_{\mu \nu}\right]\right)=E^{j} t_{j}=0$

reduce to only one Skyrme field equation (since the third Skyrme equation is identically satisfied while the first and the second are proportional):

$E^{3}=0$,

$E^{1}=I_{1} P[H], \quad E^{2}=I_{2} P[H], \quad I_{1} \neq 0, \quad I_{2} \neq 0$,

where $I_{j}$ are real and non-vanishing. Thus, the Skyrme field equations reduce to $P[H]=0$ namely

$$
\begin{aligned}
& 4\left[X \sin ^{2}(H)-\lambda\left(l_{2}^{2} q^{2}+l_{3}^{2} p^{2}\right)-4 l_{2}^{2} l_{3}^{2}\right] H^{\prime \prime} \\
& +2 X \sin (2 H) H^{\prime 2}+4 \sin ^{2}(H) X^{\prime} H^{\prime} \\
& +\left[\lambda \kappa ( l _ { 3 } ^ { 2 } p b _ { 2 } + l _ { 2 } ^ { 2 } q b _ { 3 } ) \left(-\frac{4 l_{1}^{2} p}{l_{2}^{2}} \kappa b_{2}-\frac{4 l_{1}^{2} q}{l_{3}^{2}} \kappa b_{3}\right.\right. \\
& \left.+2 l_{1}^{2}\left(\frac{q^{2}}{l_{3}^{2}}-\frac{p^{2}}{l_{2}^{2}}\right)\right)-\frac{1}{4} l_{1}^{2} X\left(\frac{p^{2}}{l_{2}^{2}}+\frac{q^{2}}{l_{3}^{2}}\right) \\
& \left.+\lambda l_{1}^{2} p^{2} q^{2}\right] \sin (4 H)-\frac{2 l_{1}^{2}}{\lambda} X \sin (2 H)=P[H]=0,
\end{aligned}
$$

where

$$
\begin{aligned}
X(r)=8 \lambda & \kappa\left(2 l_{2}^{2} l_{3}^{2} \kappa b_{1}^{2}-l_{3}^{2} b_{2}\left(2 \kappa b_{2}+p\right)\right. \\
+ & \left.l_{2}^{2} b_{3}\left(q-2 \kappa b_{3}\right)\right) .
\end{aligned}
$$

Quite remarkably, if we demand that

$$
\begin{aligned}
& X(r)=\lambda\left(l_{2}^{2} q^{2}+l_{3}^{2} p^{2}\right), \\
& b_{2}(r)=-\frac{l_{2}^{2} q}{l_{3}^{2} p} b_{3}+\frac{1}{\kappa}\left(\frac{l_{2}^{2} q^{2}}{4 l_{3}^{2} p}-\frac{p}{4}\right),
\end{aligned}
$$

then the equation for the profile $H(r)$ can be solved explicitly. More importantly, the above algebraic conditions in Eq. (42) are consistent with the Maxwell equations written above. Indeed, if one plugs the two algebraic conditions in Eq. (42) into the three Maxwell equations one obtains a single Maxwell equation for $b_{3}(r)$ :

$$
\begin{aligned}
b_{3}^{\prime \prime}= & \frac{\kappa K}{8 l_{2}^{2} l_{3}^{2}}\left(q-4 \kappa b_{3}\right)\left[8 \lambda l_{2}^{2} l_{3}^{2} H^{\prime 2}\right. \\
& +l_{1}^{2}\left(\lambda \cos (2 H)\left(l_{2}^{2} q^{2}+l_{3}^{2} p^{2}\right)\right. \\
& \left.\left.+l_{2}^{2}\left(8 l_{3}^{2}+\lambda q^{2}\right)+\lambda l_{3}^{2} p^{2}\right)\right] \sin ^{2}(H),
\end{aligned}
$$

while for the profile $H(r)$ we have a decoupled (from $b_{3}$ ) equation that reads

$$
\begin{aligned}
& {\left[\lambda \cos (2 H)\left(l_{2}^{2} q^{2}+l_{3}^{2} p^{2}\right)+l_{2}^{2}\left(8 l_{3}^{2}+\lambda q^{2}\right)+\lambda l_{3}^{2} p^{2}\right] H^{\prime \prime}} \\
& \quad+\left(l_{2}^{2} q^{2}+l_{3}^{2} p^{2}\right)\left(l_{1}^{2}-\lambda H^{\prime 2}\right) \sin (2 H)=0
\end{aligned}
$$

Thus, the big technical achievement of the present approach is that the three coupled gauged Skyrme equations in Eq. (5) and the corresponding four Maxwell equations in Eq. (6) with exactly the Skyrme ansatz in Eqs. (12) and (13) and the gauge potential in Eq. (38) reduce to Eqs. (43) and (44) when the two algebraic conditions in Eq. (42) are satisfied. We want to stress that the aforementioned relations provide an exact solution and they are not a product of an approximation. As for the boundary conditions that are needed to be set, we have to keep in mind that the system is confined to a finite box. Thus, the easiest way to realize this is by imposing periodic boundary conditions in $\gamma$ and $\phi$ and Dirichlet in $r$

Interestingly enough, Eq. (44) can be solved explicitly by observing that it has the following first integral

$Y(H) \frac{H^{\prime 2}}{2}+V(H)=E_{0}$,

with

$$
\begin{aligned}
& Y(H)=2 \lambda\left(l_{2}^{2} q^{2}+l_{3}^{2} p^{2}\right) \cos ^{2}(H)+8 l_{2}^{2} l_{3}^{2}, \\
& V(H)=-\frac{1}{2} l_{1}^{2}\left(l_{2}^{2} q^{2}+l_{3}^{2} p^{2}\right) \cos (2 H)
\end{aligned}
$$

and where $E_{0}$ is an integration constant to be determined by requiring that the boundary conditions to have nonvanishing topological charge are satisfied. Thus, Eq. (44) can be reduced to a quadrature (which defines a generalized elliptic integral). Equation (43) for $b_{3}$ is linear (since $H(r)$ can be found explicitly), however its integration is not a trivial task. In any case, integration of (43) that results in an expression for $b_{3}$ makes trivial the determination of the other two components of $A_{\mu}$ since both $b_{1}$ and $b_{2}$ are given algebraically in terms of $b_{3}$ through conditions (42). Nevertheless, even without the explicit expressions, it is still possible to analyze the generic features of the transport properties electrons passing through the above gauged Skyrmions.

\subsection{Gauged time-crystals}

In order to have a time periodic solution with a non vanishing topological charge, that can be characterized as a time-crystal (for the introduction to the notion of time crystals see [6770]) we start by considering the line element

$d s^{2}=-d \gamma^{2}+l_{1} d r^{2}+l_{2} d z^{2}+l_{3} d \phi^{2}$,

where $\gamma$ in the new ansatz

$G=\frac{q \phi-\omega \gamma}{2}, \quad A=-\frac{q \phi+\omega \gamma}{2}$ 
is the time variable, making the ensuing solution a time periodic configuration. The constant $\omega$ is the frequency of the time-crystal characterizing the periodicity of the system. Again we consider a finite box, where this time we take

$0 \leq r \leq 2 \pi, \quad 0 \leq z \leq 4 \pi, \quad 0 \leq \phi \leq 2 \pi$.

We adopt a similar form for the electromagnetic potential as the one given in (38). However, we have to note now that the index of the coordinates is changed into $x^{\mu}=(\gamma, r, z, \phi)$. Thus, the vector potential is

$$
A_{\mu}=\left(b_{2}(r), 0, b_{1}(r), b_{3}(r)\right),
$$

making $b_{2}(r)$ the electrostatic potential instead of $b_{1}(r)$ that we had in the Skyrmion case. The Maxwell equations (6) retain same form as (39) with

$$
\begin{aligned}
M_{11}= & -\frac{K}{2 l_{3}^{2}} \sin ^{2}(H)\left[8 \lambda l_{3}^{2} H^{\prime 2}\right. \\
& \left.\quad+l_{1}^{2}\left(2 \lambda \cos ^{2}(H)\left(q^{2}-l_{3}^{2} \omega^{2}\right)+8 l_{3}^{2}\right)\right] \\
M_{23}= & \frac{K \lambda l_{1}^{2} q \omega}{4 l_{3}^{2}} \sin ^{2}(2 H) \\
M_{32}= & -l_{3}^{2} M_{23} \\
M_{22}= & M_{11}+\frac{\omega}{q} M_{3,2} \\
M_{33}= & M_{11}+\frac{q}{\omega} M_{2,3} \\
N_{2}= & \frac{\omega}{4} M_{11}-\frac{1}{4}\left(\frac{l_{3}^{2} \omega^{2}}{q}+q\right) \\
N_{3}= & -\frac{q}{4} M_{11}+\frac{1}{4}\left(\frac{q^{2}}{l_{3}^{2} \omega}+\omega\right),
\end{aligned}
$$

while the rest of the components of $M$ and $N$ are zero.

As also happened in the Skyrme case, again here, the field equations reduce to a single ordinary differential equation for the profile function $H(r)$. In this case the relative equation reads

$$
\begin{aligned}
4 & \left(X \sin ^{2}(H)+l_{2}^{2}\left(l_{3}^{2}\left(\lambda \omega^{2}-4\right)-\lambda q^{2}\right)\right) H^{\prime \prime} \\
& +2 X \sin (2 H)\left(H^{\prime}\right)^{2}+4 \sin ^{2}(H) X^{\prime} H^{\prime} \\
& +\frac{l_{1}^{2}}{4 l_{3}^{2}}\left[4 \lambda l_{2}^{2}\left(2 \kappa q b_{3}-l_{3}^{2} \omega\left(2 \kappa b_{2}+\omega\right)\right)\right. \\
& \left.\times\left(2 \kappa l_{3}^{2} \omega b_{2}+q\left(q-2 \kappa b_{3}\right)\right)-X\left(q^{2}-l_{3}^{2} \omega^{2}\right)\right] \sin (4 H) \\
& -\frac{\left(2 l_{1}^{2}\right)}{\lambda} X \sin (2 H)=0,
\end{aligned}
$$

where

$$
\begin{aligned}
X(r)=- & 8 \kappa \lambda\left[2 \kappa l_{3}^{2} b_{1}^{2}\right. \\
& \left.-l_{2}^{2}\left(l_{3}^{2} b_{2}\left(2 \kappa b_{2}+\omega\right)+b_{3}\left(q-2 \kappa b_{3}\right)\right)\right] .
\end{aligned}
$$

Once more, profile equation (52) can be reduced to an integrable one that is decoupled from the Maxwell field. Let us assume the following conditions for the components $b_{1}$ and $b_{3}$ of the electromagnetic potential $A_{\mu}$ :

$X(r)=\lambda l_{2}^{2}\left(q^{2}-l_{3}^{2} \omega^{2}\right), \quad b_{3}(r)=\frac{l_{3}^{2} \omega}{q} b_{2}(r)+\frac{l_{3}^{2} \omega^{2}}{4 \kappa q}+\frac{q}{4 \kappa}$.

Then, the remaining Maxwell equation that needs to be satisfied for $b_{2}$ is

$$
\begin{aligned}
b_{2}^{\prime \prime}=- & \frac{\kappa K}{8 l_{3}^{2}}\left(4 \kappa b_{2}+\omega\right)\left[8 l_{3}^{2}\left(\lambda\left(H^{\prime}\right)^{2}+l_{1}^{2}\right)\right. \\
& \left.+2 \lambda l_{1}^{2} \cos ^{2}(H)\left(q^{2}-l_{3}^{2} \omega^{2}\right)\right] \sin ^{2}(H)
\end{aligned}
$$

and the profile equation is reduced to

$$
\begin{aligned}
& \left(2 \lambda \cos ^{2}(H)\left(q^{2}-l_{3}^{2} \omega^{2}\right)+8 l_{3}^{2}\right) H^{\prime \prime} \\
& \quad+\sin (2 H)\left(q^{2}-l_{3}^{2} \omega^{2}\right)\left(l_{1}^{2}-\lambda H^{\prime 2}\right)=0 .
\end{aligned}
$$

Obviously it exhibits a first integral of the form (45) where now

$$
\begin{aligned}
& Y(H)=2 \lambda \cos ^{2}(H)\left(q^{2}-l_{3}^{2} \omega^{2}\right)+8 l_{3}^{2} \\
& V(H)=\frac{l_{1}^{2}}{2}\left(l_{3}^{2} \omega^{2}-q^{2}\right) \cos (2 H) .
\end{aligned}
$$

We can notice the similarities with the expressions derived for the Skyrmion in the previous case. In [65] there has been presented an extensive discussion on the "extended duality" that exists between two such systems.

\subsection{Topological current for the gauged Skyrmion}

The topological current [21] of the gauged Skyrme model can be divided into two terms

$J_{\mu}^{B}=J_{\mu}^{S k}+J_{\mu}^{B-e m}$

with the first term $J_{\mu}^{S k}$ being the usual Baryonic current, while second term is the correction to the latter, owed to the coupling with the electromagnetic field. For the first term we have

$J_{\mu}^{S k}=\frac{1}{24 \pi^{2}} E_{\mu \alpha \beta \nu} \operatorname{Tr}\left(R^{\alpha} R^{\beta} R^{\nu}\right)$,

which in our case has a single nonzero component

$$
\begin{aligned}
J_{0}^{S k} & =-\frac{p q}{8 \pi^{2} l_{1} l_{2} l_{3}} H^{\prime} \sin (2 H)=-2 \pi \widehat{n}_{B} H^{\prime} \sin (2 H), \\
V & =16 \pi^{3} l_{1} l_{2} l_{3},
\end{aligned}
$$


where $V=16 \pi^{3} l_{1} l_{2} l_{3}$ is the volume of the box and $\widehat{n}_{B}$ is the Baryon density $\left(\widehat{n}_{B}=p q / V\right)$ of the system. Note that in (58) we make use of the Levi-Civita tensor $E_{\mu \alpha \beta \nu}=\sqrt{-g} \epsilon_{\mu \alpha \beta \nu}$ instead of the Levi-Civita symbol $\epsilon_{\mu \alpha \beta \nu}$ so that $J_{\mu}^{S k}$ transforms covariantly and the topological charge results in a pure number. If for instance we apply the boundary conditions $H(0)=0, H(2 \pi)=\frac{\pi}{2}$ we obtain

$B=\int_{\Sigma} \sqrt{-g} J_{S k}^{0} d r d \gamma d \phi=p q$.

The correction $J_{\mu}^{B-e m}$ to the baryonic current, due to the electromagnetic field, is

$$
\begin{aligned}
J_{\mu}^{e m}= & -\frac{\kappa}{8 \pi^{2}} E_{\mu \alpha \beta \nu} \nabla^{\alpha} \\
& \times\left[A^{\beta} \operatorname{Tr}\left(t_{3}\left(U^{-1} \nabla^{v} U-\nabla^{v} U U^{-1}\right)\right)\right]
\end{aligned}
$$

and the total gauged Baryonic current reads

$$
\begin{aligned}
J_{\mu}^{B}= & \left\{-\frac{p q \pi}{V} \partial_{r}(\cos (2 H))\right. \\
& +\frac{4 \pi \kappa}{V} \partial_{r}\left(\cos ^{2}(H)\left(q b_{2}-p b_{3}\right)\right), 0, \\
& \left.-\frac{4 \pi q \kappa}{V} \partial_{r}\left(b_{1} \cos ^{2}(H)\right), \frac{4 \pi p \kappa}{V} \partial_{r}\left(\cos ^{2}(H)\right)\right\} .
\end{aligned}
$$

From what we see, the total baryon number when the Skyrmion is coupled to the electromagnetic field depends also on the boundary conditions that one may impose on the latter ( $b_{2}$ and $b_{3}$ in particular).

\subsection{Baryonic current for the time-crystal}

The topological current of the time-crystal can be calculated with the use of the same relations (58) and (61). Here we just give the result for the full current of the Gauged Time Crystal (GTC) which is

$$
\begin{aligned}
J_{\mu}^{G T C}= & \left\{-\frac{4 \pi q \kappa}{V} \partial_{r}\left(b_{1} \cos ^{2}(H)\right), 0,\right. \\
& -\frac{l_{2}^{2} q \pi \omega}{V} \partial_{r}(\cos (2 H)) \\
& +\frac{4 \pi \kappa}{V} \partial_{r}\left[\cos ^{2}(H)\left(q b_{2}-\omega b_{3}\right)\right], \\
& \left.\frac{4 \pi \kappa \omega}{V} \partial_{r}\left(b_{1} \cos ^{2}(H)\right)\right\} .
\end{aligned}
$$

In the absence of the coupling with the electromagnetic field, $\kappa=0$, we can see that the expression for the non-zero topological current of the time-crystal is simplified to

$$
J_{\mu}^{T C}=\left\{0,0,-\frac{\pi l_{2}^{2} q \omega}{V} \partial_{r}(\cos (2 H)), 0\right\} .
$$

\section{On the conductivity of gauged solitons}

At semi-classical level, the transport properties of electrons travelling through the above gauged Skyrmions can be determined by analyzing the corresponding Dirac equation. Obviously, the electrons interact directly both with the gauge field and with the Baryons. The fermion couples to $A_{\mu}$, as QED dictates. However, there are further effects due to the coupling with the baryonic current. Here, we follow a very simple toy model interaction just to make a qualitative description of such effects. At this level of approximation in which the electrons perceive the gauged Skyrmions as a classical background, both interactions can be described as "currentcurrent" interactions in the Dirac Hamiltonian. The interaction of the electronic Dirac field $\Psi$ with the gauge potential $A_{\mu}$ corresponds to the following interaction Hamiltonian

$$
\begin{aligned}
H_{i n t}^{U(1)} & =\kappa J_{\mu}^{e} A^{\mu}, \\
J_{\mu}^{e} & =\bar{\Psi} \gamma_{\mu} \Psi, \\
\bar{\Psi} & =\Psi^{\dagger} \gamma^{0},
\end{aligned}
$$

where $\kappa$ is the Maxwell coupling

$\kappa \approx\left(\frac{1}{137}\right)^{\frac{1}{2}}$

$\gamma_{\mu}$ are the Dirac gamma-matrices (the conventions are collected in the Appendix A), $\Psi^{\dagger}$ is the conjugate transpose of $\Psi$ and $\bar{\Psi}$ the adjoint spinor. On the other hand, a simple way to describe the interactions of the electronic Dirac field with the baryonic current $J_{B}^{\mu}$ is with the following Hamiltonian

$H_{i n t}^{B}=g_{e f f} J_{\mu}^{e} J_{B}^{\mu}$,

where $g_{\text {eff }}$ is the effective coupling constant of the electronBaryon interaction. At the present level of approximation (in which the energy scale is not high enough to disclose the parton structure of the Baryon) a reasonable assumption is:

$g_{\text {eff }} \approx G_{F}$,

where $G_{F}$ is the Fermi constant.

In order to evaluate the relative strength of the two contributions to the conductivity (a brief analysis is given in Appendix B), one arising from the term owed to the coupling with the $U$ (1) field (the $\kappa A_{\mu}$ in Eq. (69), see Sect. B.1 of Appendix B) and the other arising from the term produced from the baryon current (the $G_{F} J_{\mu}^{B}$ in Eq. (69)) one needs to evaluate the relative strength of the $U(1)$ coupling with respect to the interactions with the Skyrmionic current. There are two competing factors in the interactions with the Skyrmionic current. The first factor is the electro-weak coupling constant (which is obviously weaker than the $U(1)$ coupling). The second factor is related with the Skyrmions 
profile $H$ and can be evaluated explicitly thanks to the present analytic solutions. Assuming that both $\sin (2 H)$ and $H^{\prime}$ are of order 1 (since both quantities are adimensional and the solitonic solutions we are considering are smooth and regular) one can see that the effective adimensional coupling $\widehat{g}$ measuring the strength of the contributions to the conductivity due to the interactions of the electrons with the Skyrmionic current is:

$\widehat{g}=l_{1} G_{F} \widehat{n}_{B}$.

Given that $G_{F} \sim 1.166 \mathrm{GeV}^{-2}$ or $G_{F} \sim 4.564 \mathrm{fm}^{2}$ in natural units we can see that the contribution of the interaction with $J_{\mu}^{B}$ remains small in comparison to the coupling with $A_{\mu}$ - at least for baryon densities $\widehat{n}_{B}$ and lengths $l_{1}$ of the box that can be characterized as natural. The "Baryonic" correction $\delta \Psi$ to the wave function in Eq. (75) depends on the effective coupling $\widehat{g}$ defined in Eq. (68) and on the Fourier transform of quantities related with the background Skyrmion.

For completeness, in Sects. B.2 and B.3 of Appendix B we have included the Dirac equations for the electrons propagating in the gauged solitons background described above. Although these Dirac equations cannot be solved analytically (due to the fact that Eqs. (43) and (55) are not integrable in general), they can be useful starting points for numerical analysis of transport properties of the present gauged solitons.

\section{Conclusions and perspectives}

In the present paper we have studied (gauged) Skyrmionic configurations in a finite box. We provided the reduced field equations under the adopted ansatz and distinguished the conditions over the potential functions $A_{\mu}$ for which the aforementioned equations can be characterized as integrable. Additionally, we have presented analytic expressions for the energy and studied its general behaviour in relation to the baryon number and the possible sizes of the box under consideration. We also managed to demonstrate and analyze the cases where the more energetically convenient configurations emerge in relations to these variables.

What is more, we have derived an explicit analytic expression for the compression modulus corresponding to Skyrmions living within a finite volume in flat space-times. This is the first case in which one can derive an analytic formula (Eqs. (36) and (37) in the previous section) for such an important quantity in a highly interacting theory such as the low energy limit of QCD. This expression produces a reasonable value with a correct order of magnitude. The gauged version of these solitons living within a finite volume can be also considered. Using these gauged solitons, it is possible to analyze the contributions to the electrons conductivity associated to the interactions with this Baryonic environment (which represents a slab of baryons which can be very large in two of the three spatial directions). To the best of authors knowledge, the present is the first concrete setting in which it is possible to perform analytic computations of these relevant quantities in the original version of the Skyrme model (and its gauged version).

Acknowledgements The authors would like to thank A. Zerwekh for useful discussions. This work has been funded by the Fondecyt grants 1160137 and 3160121. The Centro de Estudios Científicos (CECs) is funded by the Chilean Government through the Centers of Excellence Base Financing Program of Conicyt.

Data Availability Statement This manuscript has no associated data or the data will not be deposited. [Authors' comment: This is a theoretical work. No experimental data where used.]

Open Access This article is distributed under the terms of the Creative Commons Attribution 4.0 International License (http://creativecomm ons.org/licenses/by/4.0/), which permits unrestricted use, distribution, and reproduction in any medium, provided you give appropriate credit to the original author(s) and the source, provide a link to the Creative Commons license, and indicate if changes were made.

Funded by $\mathrm{SCOAP}^{3}$.

\section{Appendix A: Conventions}

Throughout the paper we use the metric signature $(-,+$, $+,+)$. The ordering of the space-time coordinates is $x^{\mu}=$ $(z, r, \gamma, \phi)$ for the Skyrmion and $x^{\mu}=(\gamma, r, z, \phi)$ for the time-crystal.

The four Dirac matrices are

$$
\begin{aligned}
& \gamma^{0}=\left(\begin{array}{cccc}
1 & 0 & 0 & 0 \\
0 & 1 & 0 & 0 \\
0 & 0 & -1 & 0 \\
0 & 0 & 0 & -1
\end{array}\right), \quad \gamma^{1}=\left(\begin{array}{cccc}
0 & 0 & 0 & 1 \\
0 & 0 & 1 & 0 \\
0 & -1 & 0 & 0 \\
-1 & 0 & 0 & 0
\end{array}\right) \\
& \gamma^{2}=\left(\begin{array}{cccc}
0 & 0 & 0 & -\dot{\mathbf{i}} \\
0 & 0 & \dot{\mathbf{i}} & 0 \\
0 & \dot{\boldsymbol{i}} & 0 & 0 \\
-\dot{\mathbf{i}} & 0 & 0 & 0
\end{array}\right), \quad \gamma^{3}=\left(\begin{array}{cccc}
0 & 0 & 1 & 0 \\
0 & 0 & 0 & -1 \\
-1 & 0 & 0 & 0 \\
0 & 1 & 0 & 0
\end{array}\right) \text {. }
\end{aligned}
$$

\section{Appendix B: Dirac equation}

Here we include, for completeness, the Dirac equation for an electron propagating in the two gauged solitons described in the main text. Although, in these cases, the Dirac equation cannot be solved analytically, it shows clearly that the present framework provides with a concrete setting to attack computations which, at a first glance, could appear very difficult (like the conductivities associated to gauged solitons at finite densities). 


\section{B.1 Qualitative analysis}

The Dirac equation which describes the propagation of the electron through the above gauged Skyrmion is

$\left[\gamma^{\mu}\left(\dot{\mathrm{I}} \nabla_{\mu}-\kappa A_{\mu}-G_{F} J_{\mu}^{B}\right)+m\right] \Psi(z, r, \gamma, \phi)=0$,

where $m$ is the electron mass and $J_{\mu}^{B}$ is given by (62). It is convenient to write the above Dirac equation as follows ${ }^{5}$ :

$\left(H_{0}+H_{\text {int }}\right) \Psi=0$,

$H_{0}=\left[\dot{\mathrm{i}} \gamma^{\mu} \nabla_{\mu}+m\right]$,

$H_{\text {int }}=\left[\gamma^{\mu}\left(-\kappa A_{\mu}-G_{F} J_{\mu}^{B}\right)\right]$.

We will work to first order in perturbation theory and we will consider $H_{\text {int }}$ as a small perturbation. The main goal of our analysis is to take the first order corrections to the conductivity and make a comparison between the part that is owed to the interactions with the solitons and the usual contributions arising from electromagnetic sources other than the soliton itself.

The last ingredient we need is the Kubo formula for the conductivity associated to electrons moving in a medium (for a detailed review see chapter 4 of [77]). Following the usual steps one arrives at the following expression for the conductivity $\sigma_{\mu \nu}(\vec{q}, \Omega)$ (where $\vec{q}$ and $\Omega$ the wave vector and frequency respectively of the incident electromagnetic wave):

$$
\begin{aligned}
\sigma_{\mu \nu}(\vec{q}, \Omega)= & \sum_{s} \frac{1}{\hbar \Omega} \int d t\langle s| J_{0 \mu}^{e}(\vec{q}, 0) \\
& \times J_{0 v}^{* e}(\vec{q}, \Omega)|s\rangle \exp [-i \Omega t]
\end{aligned}
$$

where $|s\rangle$ and $J_{0 \mu}^{e}$ are the eigenstate of the free Dirac Hamiltonian and the corresponding current in the box where the gauged solitons live.

Due to the interaction Hamiltonian $H_{\text {int }}$ defined $^{6}$ in Eqs. (70)-(72), the electron currents $J_{\mu}^{e}=\bar{\Psi} \gamma_{\mu} \Psi$ changes

$J_{0 \mu}^{e} \rightarrow J_{0 \mu}^{e}+(\delta \bar{\Psi}) \gamma_{\mu} \Psi+\bar{\Psi} \gamma_{\mu}(\delta \Psi)=J_{0 \mu}^{e}+\delta J_{\mu}^{e}$,

where $\delta \Psi$ can be computed using first order perturbation theory. In particular, if $\Psi_{0}$ is a solution of the un-perturbed equation

$H_{0} \Psi_{0}=E \Psi_{0}$,

\footnotetext{
$\overline{5}$ On the other hand, the gauge potential $A_{\mu}$ and the Baryonic current $J_{\mu}^{B}$ are the ones corresponding to the gauged Skyrmion and gauged time-crystal described in the previous section.

6 The gauge potential $A_{\mu}$ and the Baryon current $J_{\mu}^{B}$ in the interaction Hamiltonian are the ones corresponding to the gauged Skyrmion and to the gauged time-crystal defined in the previous section.
}

then the eigenstate $\Psi$ of the interacting case can be written as

$\Psi=\Psi_{0}-H_{0}^{-1}\left(H_{i n t} \Psi_{0}\right)$,

where $H_{0}^{-1}$ is the inverse Dirac operator defined as the Green function $H_{0}^{-1}=G\left(x-x^{\prime}\right)$ satisfying

$H_{0} G_{0}\left(x-x^{\prime}\right)=\delta\left(x-x^{\prime}\right)$.

We now from the free particle case that the Green function in space-time variables is expressed as

$H_{0}^{-1}=G_{0}\left(x-x^{\prime}\right)=\int \frac{d^{4} k}{(2 \pi)^{4}} e^{-\dot{\mathrm{i}} k_{\mu}\left(x^{\mu}-x^{\prime \mu}\right)} \frac{m-\gamma^{\mu} k_{\mu}}{k_{\mu} k^{\mu}+m^{2}}$

(of course in our case, for the finite box, the integral is to be substituted by series). Consequently, we have a perturbation of the form

$$
\begin{aligned}
\delta \Psi= & \int \frac{d^{4} k}{(2 \pi)^{4}} \int d^{4} x^{\prime-\dot{i} k_{\mu}\left(x^{\mu}-x^{\prime \mu}\right)} \frac{m-\gamma^{\mu} k_{\mu}}{k_{\mu} k^{\mu}+m^{2}} \\
& \times\left(\kappa A_{\mu}+G_{F} J_{\mu}^{B}\right) \Psi_{0}\left(x^{\prime}\right)
\end{aligned}
$$

owed to two contributions; the Maxwell field $A_{\mu}$ and the baryon current $J_{\mu}^{B}$.

As for the free particle solution $\Psi_{0}$, it is easy to see that

$\Psi_{0}(x)=\left(\begin{array}{l}\psi_{1} \\ \psi_{2} \\ \psi_{3} \\ \psi_{4}\end{array}\right) e^{-\dot{i} k_{\mu} x^{\mu}}$

with

$\psi_{1}=\frac{k_{3} \psi_{3}+\left(k_{1}-\dot{i} k_{2}\right) \psi_{4}}{k_{0}+m}$,

$\psi_{2}=\frac{\left(k_{1}+\dot{i} k_{2}\right) \psi_{3}-k_{3} \psi_{4}}{k_{0}+m}, \quad k_{0}^{2}=\vec{k}^{2}+m$

satisfies $H_{0} \Psi_{0}=0$.

Consequently,

$$
\begin{aligned}
\sigma_{\mu \nu} \rightarrow & \sigma_{\mu \nu}+\delta \sigma_{\mu \nu}, \\
\delta \sigma_{\mu \nu}= & \sum_{s} \frac{1}{\hbar \Omega} \int d t\langle s|\left[\delta J_{0 \mu}^{e}(\vec{q}, 0) J_{0 v}^{* e}(\vec{q}, \Omega)\right. \\
& \left.+J_{0 \mu}^{e}(\vec{q}, 0) \delta J_{0 v}^{* e}(\vec{q}, \Omega)\right]|s\rangle \exp [-i \Omega t] .
\end{aligned}
$$

\section{B.2 Dirac equation for the gauged Skyrmion}

The symmetries of the problem allow to search for a separated solution of the form 


$$
\begin{aligned}
& \Psi(z, r, \gamma, \phi) \\
& \quad=e^{-\dot{i}\left(\omega_{1} z-k_{2} \gamma-k_{3} \phi\right)}\left\{\psi_{1}(r), \psi_{2}(r), \psi_{3}(r), \psi_{4}(r)\right\} .
\end{aligned}
$$

By introducing (78) into the Dirac equation (69) we obtain the following set of equations for the components of $\Psi$ :

$$
\begin{aligned}
\psi_{1}^{\prime}= & \left(k_{2}+\kappa b_{2}-\kappa g \frac{q}{4 \pi^{2} V} \phi_{1}^{\prime}\right) \psi_{1} \\
& +\dot{\mathbf{I}}\left(k_{3}+\kappa b_{3}+\kappa g \frac{p}{4 \pi^{2} V} \phi_{1}^{\prime}\right) \psi_{2} \\
& +\dot{\mathbf{I}}\left(\omega_{1}-m-\kappa b_{1}-\frac{g}{16 \pi^{2} V}\left(4 \kappa \phi_{2}^{\prime}+\phi_{3}^{\prime}\right)\right) \psi_{4}
\end{aligned}
$$

$$
\begin{aligned}
\psi_{2}^{\prime}= & -\dot{\mathbf{I}}\left(\kappa b_{3}+k_{3}+\kappa g \frac{p}{4 \pi^{2} V} \phi_{1}^{\prime}\right) \psi_{1} \\
& -\left(\kappa b_{2}+k_{2}-\kappa g \frac{q}{4 \pi^{2} V} \phi_{1}^{\prime}\right) \psi_{2} \\
& +\dot{\mathbf{I}}\left(\omega_{1}-m-\kappa b_{1}-\frac{g}{16 \pi^{2} V}\left(4 \kappa \phi_{2}^{\prime}+\phi_{3}^{\prime}\right)\right) \psi_{3}
\end{aligned}
$$

$$
\begin{aligned}
\psi_{3}^{\prime}= & \dot{\mathbf{i}}\left(\omega_{1}+m-\kappa b_{1}-\frac{g}{16 \pi^{2} V}\left(4 \kappa \phi_{2}^{\prime}+\phi_{3}^{\prime}\right)\right) \psi_{2} \\
& +\left(k_{2}+\kappa b_{2}-\kappa g \frac{q}{4 \pi^{2} V} \phi_{1}^{\prime}\right) \psi_{3} \\
& +\dot{\mathbf{I}}\left(k_{3}+\sigma b_{3}+\kappa g \frac{p}{4 \pi^{2} V} \phi_{1}^{\prime}\right) \psi_{4}
\end{aligned}
$$$$
\psi_{4}^{\prime}=\dot{\underline{1}}\left(\omega_{1}+m-\kappa b_{1}-\frac{g}{16 \pi^{2} V}\left(4 \kappa \phi_{2}^{\prime}+\phi_{3}^{\prime}\right)\right) \psi_{1}
$$$$
-\dot{\mathbf{I}}\left(\kappa b_{3}+k_{3}+\kappa g \frac{p}{4 \pi^{2} V} \phi_{1}^{\prime}\right) \psi_{3}
$$$$
-\left(\kappa b_{2}+k_{2}-\kappa g \frac{q}{4 \pi^{2} V} \phi_{1}^{\prime}\right) \psi_{4},
$$

where

$\phi_{1}(r)=b_{1}(r) \cos ^{2}(H(r))$,

$\phi_{2}(r)=\cos ^{2}(H(r))\left(q b_{2}(r)-p b_{3}(r)\right)$,

$\phi_{3}(r)=p q \cos (2 H(r))$.

\section{B.3 Dirac equation for the gauged time-crystal}

By using the expression for $J_{\mu}^{G T C}$ as given by (63) inside (69), instead of $J_{\mu}^{B}$ that we had for the Skyrmion, and by considering a separable solution of the form

$$
\begin{aligned}
& \Psi(z, r, \gamma, \phi) \\
& \quad=e^{-\dot{i}\left(\omega_{1} \gamma-k_{2} z-k_{3} \phi\right)}\left\{\psi_{1}(r), \psi_{2}(r), \psi_{3}(r), \psi_{4}(r)\right\}
\end{aligned}
$$

we obtain a system of equations given by

$$
\begin{aligned}
\psi_{1}^{\prime}= & \left(\kappa b_{2}-\omega_{1}-\frac{g q \kappa}{4 \pi^{2} V} \phi_{1}^{\prime}\right) \psi_{1} \\
& +\dot{\mathbf{i}}\left(\kappa b_{3}+k_{3}+\frac{g \kappa \omega}{4 \pi^{2} V} \phi_{1}^{\prime}\right) \psi_{2} \\
& -\dot{\mathrm{i}}\left(\kappa b_{1}+k_{2}+m+\frac{g}{16 \pi^{2} V}\left(4 \kappa \phi_{2}^{\prime}-l_{2}^{2} \phi_{3}^{\prime}\right)\right) \psi_{4}
\end{aligned}
$$

$$
\begin{aligned}
\psi_{2}^{\prime}= & -\dot{\mathbf{i}}\left(\kappa b_{2}+k_{3}+\frac{g \kappa \omega}{4 \pi^{2} V} \phi_{1}^{\prime}\right) \psi_{1} \\
& +\left(\omega_{1}-\kappa b_{2}+\frac{g q \kappa}{4 \pi^{2} V} \phi_{1}^{\prime}\right) \psi_{2} \\
& -\dot{\mathbf{i}}\left(\kappa b_{1}+k_{2}+m+\frac{g}{16 \pi^{2} V}\left(4 \kappa \phi_{2}^{\prime}-l_{2}^{2} \phi_{3}^{\prime}\right)\right) \psi_{3}
\end{aligned}
$$

$$
\begin{aligned}
\psi_{3}^{\prime}= & \dot{\mathbf{i}}\left(m-\kappa b_{1}-k_{2}-\frac{g}{16 \pi^{2} V}\left(4 \kappa \phi_{2}^{\prime}-l_{2}^{2} \psi_{3}^{\prime}\right)\right) \psi_{2} \\
& \left(\kappa b_{2}-\frac{g q \kappa}{4 \pi^{2} V} \phi_{1}^{\prime}-\omega_{1}\right) \psi_{3} \\
& +\dot{\mathrm{i}}\left(\kappa b_{3}+k_{3}+\frac{g \kappa \omega}{4 \pi^{2} V} \phi_{1}^{\prime}\right) \psi_{4}
\end{aligned}
$$

$$
\begin{aligned}
\psi_{4}^{\prime}= & \dot{\mathbf{i}}\left(m-\kappa b_{1}-k_{2}-\frac{g}{16 \pi^{2} V}\left(4 \kappa \phi_{2}^{\prime}-l_{2}^{2} \phi_{3}^{\prime}\right)\right) \psi_{1} \\
& -\dot{\mathbf{i}}\left(\kappa b_{3}+k_{3}+\frac{g \kappa \omega}{4 \pi^{2} V} \phi_{1}^{\prime}\right) \psi_{3} \\
& +\left(\omega_{1}-\kappa b_{2}+\frac{g q \kappa}{4 \pi^{2} V} \phi_{1}^{\prime}\right) \psi_{4}
\end{aligned}
$$

The functions $\phi_{1}(r), \phi_{2}(r)$ and $\phi_{3}(r)$ are the same as before, only now we have $\omega$ appearing in them in place of $p$, i.e.

$$
\begin{aligned}
& \phi_{1}(r)=b_{1}(r) \cos ^{2}(H(r)) \\
& \phi_{2}(r)=\cos ^{2}(H(r))\left(q b_{2}(r)-\omega b_{3}(r)\right) \\
& \phi_{3}(r)=q \omega \cos (2 H(r)) .
\end{aligned}
$$

\section{References}

1. T. Skyrme, Proc. R. Soc. Lond. A 260, 127 (1961)

2. T. Skyrme, Proc. R. Soc. Lond. A 262, 237 (1961)

3. T. Skyrme, Nucl. Phys. 31, 556 (1962)

4. T.S. Walhout, Nucl. Phys. A 531, 596 (1991)

5. C. Adam, M. Haberichter, A. Wereszczynski, Phys. Rev. C 92, 055807 (2015)

6. J.-I. Fukuda, S. Žumer, Nat. Commun. 2, 246 (2011)

7. H. Stefan et al., Nat. Phys. 7, 713 (2011)

8. D. Fostar, S. Krusch, Nucl. Phys. B 897, 697 (2015)

9. M. Gillard, Nucl. Phys. B 895, 272 (2015)

10. E. Witten, Nucl. Phys. B 223, 422 (1983)

11. E. Witten, Nucl. Phys. B 223, 433 (1983)

12. D. Finkelstein, J. Rubinstein, J. Math. Phys. 9, 1762-1779 (1968)

13. N. Manton, P. Sutcliffe, Topological Solitons (Cambridge University Press, Cambridge, 2007)

14. V.G. Makhanov, Y.P. Rybakov, V.I. Sanyuk, The Skyrme Model (Springer, Berlin, 1993) 
15. D. Giulini, Mod. Phys. Lett. A 8, 1917-1924 (1993)

16. A.P. Balachandran, A. Barducci, F. Lizzi, V.G.J. Rodgers, A. Stern, Phys. Rev. Lett. 52, 887 (1984)

17. G.S. Adkins, C.R. Nappi, E. Witten, Nucl. Phys. B 228, 552-566 (1983)

18. E. Guadagnini, Nucl. Phys. B 236, 35-47 (1984)

19. G. Dvali, A. Gußmann, Nucl. Phys. B 913, 1001 (2016)

20. A.D. Jackson, N.S. Manton, A. Wirzba, Nucl. Phys. A 495, 499 (1989)

21. C.G. Callan Jr., E. Witten, Nucl. Phys. B 239, 161-176 (1984)

22. J.M. Gipson, H.C. Tze, Nucl. Phys. B 183, 524 (1981)

23. J. Goldstone, F. Wilczek, Phys. Rev. Lett. 47, 986 (1981)

24. E. D'Hoker, E. Farhi, Nucl. Phys. B 241, 109 (1984)

25. V.A. Rubakov, Nucl. Phys. B 256, 509 (1985)

26. B.M.A.G. Piette, D.H. Tchrakian, Phys. Rev. D 62, 025020 (2000)

27. E. Radu, D.H. Tchrakian, Phys. Lett. B 632, 109-113 (2006)

28. I. Klebanov, Nucl. Phys. B 262, 133 (1985)

29. A. Actor, Phys. Lett. B 157, 53 (1985)

30. H.A. Weldon, Phys. Rev. D 26, 1394 (1982)

31. M. Loewe, S. Mendizabal, J.C. Rojas, Phys. Lett. B 632, 512 (2006)

32. J.A. Ponciano, N.N. Scoccola, Phys. Lett. B 659, 551 (2008)

33. M. Kugler, S. Shtrikman, Phys. Rev. D 40, 3421 (1989)

34. M. Kugler, S. Shtrikman, Phys. Lett. B 208, 491 (1988)

35. M. Harada, Y.L. Ma, H.K. Lee, M. Rho, in Fractionized Skyrmions in Dense Compact-Star Matter. The Multifaceted Skyrmion, 2nd edn., ed. by M. Rho, I. Zahed (World Scientific, Singapore, 2016)

36. Y.-L. Ma, M. Rho, Effective Field Theories for Nuclei and Compact-Star Matter (World Scientific, Singapore, 2018)

37. L.C. Biedenharn, Y. Dothan, M. Tarlini, Phys. Rev. D 31, 649 (1985)

38. U.-G. Meissner, I. Zahed, Adv. Nucl. Phys. 17, 143 (1986)

39. T. Meissner, F. Grummer, K. Goeke, M. Harvey, Phys. Rev. D 39, 1903 (1989)

40. C. Adam, C. Naya, J. Sanchez-Guillen, J.M. Speight, A. Wereszczynski, Phys. Rev. D 90, 045003 (2014)

41. H. Walliser, A. Hayashi, G. Holzwarth, Nucl. Phys. A 456, 717 (1986)

42. B.E.S. Collaboration, J.Z. Bai et al., Phys. Rev. Lett. 91, 022001 (2003)

43. BES Collaboration, M. Ablikim et al., Phys. Rev. D 71, 072006 (2005). arXiv:hep-ex/0503030

44. BES Collaboration, M. Ablikim et al., Phys. Rev. Lett. 93, 112002 (2004)

45. S. Olsen, in Invited talk at the International Symposium on Hadron Spectroscopy, Chiral Symmetry and Relativistic Description of Bound States, Tokyo (2003). arXiv:hep-ex/0305048
46. Z.-G. Wang, Eur. Phys. J. A 47, 71 (2011)

47. D.G. Ravenhall, C.J. Pethick, J.R. Wilson, Phys. Rev. Lett. 50, 2066 (1983)

48. D. Page, S. Reddy, Annu. Rev. Nucl. Part. Sci. 56, 327-374 (2006)

49. W.G. Newton, Nat. Phys. 9, 396 (2013)

50. J.A. Pons, D. Viganò, N. Rea, Nat. Phys. 9, 431 (2013)

51. D.G. Yakovlev, MNRAS 453, 581 (2015)

52. F. Canfora, H. Maeda, Phys. Rev. D 87, 084049 (2013)

53. F. Canfora, Phys. Rev. D 88, 065028 (2013)

54. F. Canfora, F. Correa, J. Zanelli, Phys. Rev. D 90, 085002 (2014)

55. F. Canfora, A. Giacomini, S. Pavluchenko, Phys. Rev. D 90, 043516 (2014)

56. S. Chen, Y. Li, Y. Yang, Phys. Rev. D 89, 025007 (2014)

57. E. Ayon-Beato, F. Canfora, J. Zanelli, Phys. Lett. B 752, 201-205 (2016)

58. F. Canfora, M. Di Mauro, M.A. Kurkov, A. Naddeo, Eur. Phys. J. C 75, 443 (2015)

59. F. Canfora, G. Tallarita, Nucl. Phys. B 921, 394 (2017)

60. F. Canfora, G. Tallarita, Phys. Rev. D 94, 025037 (2016)

61. F. Canfora, G. Tallarita, Phys. Rev. D 91, 085033 (2015)

62. F. Canfora, G. Tallarita, JHEP 1409, 136 (2014)

63. F. Canfora, S.H. Oh, P. Salgado-Rebolledo, Phys. Rev. D 96, 084038 (2017)

64. P.D. Alvarez, F. Canfora, N. Dimakis, A. Paliathanasis, Phys. Lett. B 773, 401-407 (2017)

65. L. Aviles, F. Canfora, N. Dimakis, D. Hidalgo, Phys. Rev. D 96, 125005 (2017)

66. H. Weigel, Chiral Soliton Models for Baryons. Lecture Notes in Physics (Springer, Berlin, 2008)

67. F. Wilczek, Phys. Rev. Lett. 109, 160401 (2012)

68. A. Shapere, F. Wilczek, Phys. Rev. Lett. 109, 160402 (2012)

69. F. Wilczek, Phys. Rev. Lett. 111, 250402 (2013)

70. K. Sacha, J. Zakrzewski, Time crystals: a review. Rep. Prog. Phys. 81, 016401 (2018). arXiv:1704.03735

71. Y.M. Shnir, Magnetic Monopoles (Springer, Berlin, 2005), p. 500

72. G.E. Brown, E. Osnes, Phys. Lett. B 159, 223-227 (1985)

73. G. Co', J. Speth, Phys. Rev. Lett. 57, 547-550 (1986)

74. J.P. Blaizot, Phys. Rep. 64, 171-248 (1980)

75. M.E. Caplan, C.J. Horowitz, Rev. Mod. Phys. 89, 041002 (2017)

76. M. Dutra, O. Lourenço, J.S. Sà Martins, A. Delfino, J.R. Stone, P.D. Stevenson, Phys. Rev. C 85, 035201 (2012)

77. M. Dressel, G. Grüner, Electrodynamics of Solids (Cambridge University Press, Cambridge, 2002) 